\title{
Religion und ihre Einbettung in Verfassungen als Kontext
}

\author{
Annette Schnabel (D) - Lisa Hönes
}

Eingegangen: 6. August 2020 / Überarbeitet: 24. März 2021 / Angenommen: 25. April 2021 / Online publiziert: 31. Mai 2021

(C) Der/die Autor(en) 2021, korrigierte Publikation 2022

Zusammenfassung Soziologisch lassen sich Verfassungstexte als wichtiges Moment der Konstruktion kollektiver Identitäten und idealer Mitgliedschaften staatlicher Gemeinschaften verstehen. Auf der Basis einer quantitativen, explorativen Dokumentenanalyse von Verfassungstexten aus 186 Ländern wird untersucht, welche religionsbezogenen Identifikationsangebote Verfassungen machen. Dies wird anhand von drei Themenfeldern expliziert: (i) Präambeln leiten den Verfassungstext ein, hier findet sich die Bestimmung des „wir“ der verfassungsgebenden Gemeinschaft, indem sie den „Geist“, in dem die Verfassung verfasst ist, entfalten; (ii) Die Untersuchung der Verankerung von Religions- und Gewissensfreiheit sowie ihrer Einschränkungen in den Verfassungstexten gibt Auskunft über den Stellenwert, den Religion und Religiosität für die Verfasstheit der good citizens hat. Hieran wird auch sichtbar, welches Verhältnis staatlich verfasste Sozialitäten zur individuellen Religionsfreiheit haben. (iii) Staatliche Biopolitiken wiederum umfassen neben anderen policies auch die verfassungsmäßige Verankerung der gouvernementalen Verantwortung für die (Aus-)Bildung der future citizens. Diese umfasst Art und Umfang der Schulpflicht ebenso wie die Verankerung religions-bezogener Curricula. Mit ihren Identitätsangeboten konfigurieren Verfassungen den good citizen und schaffen damit eine soziale Ordnung, indem Bürger*innen als Subjekte mit Rechten und Pflichten angerufen werden. Im globalen Vergleich bleibt Religion dabei wichtiges Element sei es als Adresse der staatlichen Schutzverpflichtung individueller Freiheiten, als Moment der Bedrohung der Freiheit anderer und der staatlichen Ordnung oder als konfessionell bestimmter Bestandteil der staatlichen Gemeinschaft.

\footnotetext{
Annette Schnabel $(\bowtie) \cdot$ Lisa Hönes

Institut für Sozialwissenschaften, Heinrich-Heine-Universität Düsseldorf,

Ulenbergstr. 127, 40225 Düsseldorf, Deutschland

E-Mail: schnabel@hhu.de

Lisa Hönes

E-Mail: Lisa.Hoenes@uni-duesseldorf.de
} 
Schlüsselwörter Verfassungsvergleich $\cdot$ Religion $\cdot$ Textanalyse $\cdot$

Verfassungsidentität · Good citizen

\section{Religion and its embeddedment in constitutional texts worldwide}

Abstract Sociologically, constitutional texts can be understood as an important factor for constructing collective identities and ideal memberships of state communities. Based on a quantitative, explorative document analysis of 186 countries' constitutional texts, we examined which religion-referring offers of identification are made by constitutional texts. This is expatiated on by means of three subject fields: (i) Preambles introduce constitutional texts; it is here that the definition of "we" in the constituent community occurs by unfolding the "spirit" in which the constitution was composed; (ii) the examination of freedom of religion and conscience as well as restrictions thereof discloses the significance which religion and religiosity have for the constituted-ness of the 'good citizens'. With this, the relation of stateconstituted socialities with individual freedom of religion becomes visible as well. (iii) The biopolitics of states, in turn, encompass - next to other policies - the constitutional anchoring of governmental responsibility for educating and formating of 'future citizens'. This includes the manner and scope of compulsory schooling as well as the anchoring of religion-referring curricula. With their offers of identity, constitutions configurate the 'good citizen' and - by calling on citizens as subjects with rights and duties - thereby create a social order of the state. In global comparison, religion remains an important element of this - be it as an address of the state's obligation to individual freedoms, as a factor of threat towards the freedom of others and the state order or as a denominationally determined component of the state community.

Keywords Constitutionalism · Religion · Text analysis · Constitutional identity · Good citizen

Constitution makers must take the people as they are and, at the same moment, seek to make them something else. Tushnet (2010, S. 672)

\section{Einleitung}

Verfassungen sind sowohl formalrechtlich als auch aus soziologischer Perspektive Rechtsvorschriften der besonderen Art. Ihre rechtspositivistische Besonderheit geht zum einen auf die besonderen Verfahren ihrer Verabschiedung zurück (u. a. Öhlinger und Eberhard 2019, S. 27; Böckenförde 2011): Verfassungen werden in einem erschwerten Verfahren erlassen und genießen besondere Bestandskraft. In ihnen ist das Verfahren ihrer Abänderung hinterlegt. In der Hierarchie der staatlichen Rechtsgebung sind alle anderen Rechtsakte an sie gebunden. Zum anderen ist die Besonderheit von Verfassungen an ihre rechtlichen Qualitäten geknüpft (z. B. Tschentscher 2011, S. 3-4): Verfassungen regeln die gesellschaftliche Grundordnung und legen Staatsform und Gewaltenteilung fest. Sie ordnen und begrenzen den Einflussbereich 
des Staates und umfassen die als zentral erachteten Schutz- und Freiheitsrechte der Staatsbürger*innen.

Soziologisch sind Verfassungen jedoch mehr: Sie spiegeln die gesellschaftlichen Auseinandersetzungen um politische Machtverhältnisse und Deutungshoheiten zur Zeit ihrer Verabschiedung (z. B. Klein und Sajó 2012). ${ }^{1}$ Über ihre historische Bedeutung für die Ausdifferenzierung des Rechtssystems (Luhmann 1990) hinaus haben sie einen „eigentümlichen Bedeutungsüberschuss“ (Witte und Bucholc 2016, S. 3), indem sie Wertsetzungen, Idealvorstellungen und normativen Verortungen der sie verabschiedenden Gesellschaften inkorporieren; sie reflektieren gesellschaftliche Selbst-Verständnisse (Böckenförde 2011, S. 13 ff.; Häberle 1998). Dazu gehören auch Vorstellungen darüber, was als good citizenship gelten soll (Kymlicka und Norman 1994). Verfassungen bleiben sozial nicht folgenlos: Sie sind das gesetzliche Gerüst innerhalb dessen individuelle und korporative Akteure agieren, sie beeinflussen politische Akteure und deren Aktionsräume genauso wie das Grundverständnis der Bürger*innen über sich als Teil ihrer Gesellschaft. Mit den in ihnen enthaltenen Selbstbeschreibungen des Demos machen sie - in unterschiedlichem Maße verpflichtende - Identifikationsangebote für dessen Mitglieder.

Diese Identifikationsangebote werden insbesondere dann sichtbar, wenn Religion und Religiosität in den Blick genommen werden. Das betrifft sowohl die Verortung der kollektiven Identität des durch die Verfassung konstituierten Demos als auch die Garantie von individuellen Religions- und Gewissensfreiheiten, die Staatsbürger*innen in unterschiedlicher Art und Weise als Träger*innen und Ausführende von Religion identifizieren. Verfassungen bestimmen damit über nationale Vergemeinschaftungen jenseits der bloßen formalen Staatszugehörigkeit, indem sie die Vorstellung darüber verfassen, wie die aktuellen und zukünftigen Mitglieder dieser Vergemeinschaftung konstituiert sein sollen (Rosenfeld 2012).

Dieser Beitrag nimmt die Idee der verfassungstextlichen Identitätsangebote auf und thematisiert Verfassungen als rechtlichen Kontext sozialer Ordnungen. Der vorliegende Beitrag klammert sowohl die Entstehungsbedingen als auch die Folgen von Verfassungen aus und konzentriert sich allein auf verfassungstextlich verfasste religionsbezogene Identitätsangebote: ${ }^{2}$ Wir nehmen an, dass bereits die Verfassungstexte Auskunft darüber geben, wie staatliche Gemeinschaften ihre kollektiven Identitäten und ihre idealen Mitglieder unter Bezugnahme auf Religion konstruieren. Auf der Basis einer quantitativen text-bezogenen Dokumentenanalyse von Verfassungstexten aus 186 Ländern (zu den Daten vgl. Schnabel et al. 2020) wird im Folgenden explorativ untersucht, welche religionsbezogenen Identifikationsangebote Verfassungen ihren Bürger*innen machen. Dies soll anhand von drei Themenfeldern expliziert werden: Erstens sollen Präambeln untersucht werden, gelten sie doch als besondere Bestandteile von Verfassungstexten (Orgad 2010). Sie leiten den Verfassungs-

\footnotetext{
1 Solche Machtverhältnisse und Verhandlungen werden sichtbar, wenn soziale, politische oder ökonomische Umbrüche Verfassungsrevisionen anstoßen (für postsowjetische Länder z. B. vgl. Glaeßner 1997; Culic 2003).

2 Der Begriff des Identitätsangebots impliziert, dass Verfassungstexte zwar bindenden Charakter haben, die Verfassungswirklichkeit hiervon jedoch abweichen kann und den Text selbst zur bloßen Deklaration werden lässt. Davon, ob und in welchem Ausmaß dies der Fall ist, soll hier jedoch abstrahiert werden.
} 
text ein, entfalten den „Geist“, in dem dieser verfasst ist und leiten die Auslegung des Verfassungstextes an. Zweitens wird die Verankerung von Religions- und Gewissensfreiheit sowie ihre Einschränkung in Verfassungstexten untersucht. Sie gibt Auskunft über den Stellenwert, den Religion und Religiosität für die Verfasstheit der good citizens hat. Hieran wird auch sichtbar, wie staatlich verfasste Sozialitäten ihr Verhältnis zur individuellen Religionsfreiheit thematisieren. Drittens wird der staatlichen Biopolitik in den Verfassungstexten nachgespürt: Sie umfasst neben anderen policies auch die verfassungsmäßige Verankerung der gouvernementalen Verantwortung für die (Aus-)Bildung der future citizens. Im Vordergrund des Beitrags steht dabei die empirisch gewendete Frage, welche Ähnlichkeiten oder Differenzen die in Verfassungen fixierten gesellschaftlichen Verständnisse des Beziehungsgeflechts aus Religion und Religiosität einerseits und Staatlichkeit andererseits aufweisen. Damit soll jenseits rechtspositivistischer Lesarten ein Beitrag zum Verständnis der identitätsschöpfenden Funktionen von Verfassungen geleistet werden.

\section{Theoretische Perspektivierung}

Aus soziologischer Perspektive sind Gesetzestexte nicht zuletzt deshalb interessant, weil sie als besondere Form der Normgebung menschliches Miteinander regeln; sie machen den justitiablen Teil der sozialen Ordnung aus, der dadurch eine besondere Verbindlichkeit erlangt. Verfassungen wiederum begründen eine besondere Form solcher gesetzesförmigen Normsetzung und sind somit mit gesellschaftlichen Vorstellungen, Konflikten und Wertvorstellungen eng verwoben (z. B. Go 2003; Thornhill 2011; Witte und Bucholc 2016).

\subsection{Verfassungen und ihre soziologische Relevanz}

Verfassungen sind eine moderne Erscheinung und haben sich historisch im Laufe der Entstehung moderner Staatlichkeit entwickelt. Der Verfassungsrechtler Blaustein bemerkt hierzu: ,[w] live in the age of constitutionalism“ (Blaustein 1994, S v); auch nach wie vor gelten Verfassungen als Ausdruck von Modernität und rationaler Staatlichkeit. Verfassungen haben damit den Status der best practice der Staats(be)gründung (Boli-Bennett 1979; Lutz 2000; Thornhill 2011). Dies macht sie auch soziologisch relevant.

Formal sind Verfassungen Normen, die ,put down in writing“ (Grey 1984, S. 15), sie sind also zeichenförmig stabilisiert, verfestigt und generalisiert. ${ }^{3}$ Verfassungen sind fundamentales Recht und werden im Vollzug damit anderen staatlichen Rechtsgebungsverfahren übergeordnet, sie umfassen aber keine explizit formulierten Sanktionen (Tschentscher 2011, S. 3 f.; vgl. auch Gardbaum 2012, S. 173 f.; Böckenförde 2011). Sie werden in andere Gesetzestexte, Regularien und policies übersetzt und sind ,institutions grounding institutions“ (Schnabel et al. 2017, S. 556). Sie sind

\footnotetext{
3 Verschriftlichung innerhalb eines expliziten Verfassungstextes ist allerdings keine notwenige Bedingung für den Verfassungscharakter einer Norm; auch abgeleitete Rechtsnormen können Verfassungscharakter haben (Gamper 2012, S. 7), ebenso Reglementierungen, Reden oder Gebräuche (Go 2003, S. 72).
} 
das Ergebnis von verfassungsgebenden Verfahren, die oft konfliktiv verlaufen und die sich in ihrer verabschiedeten Form als immer vorläufigen Konsens darüber lesen lassen, wie das politische und auch soziale Miteinander einer Gesellschaft geordnet sein soll; damit sind sie ,the web of society's basic institutions and ideals“ (Grey 1984, S. 16), das konstant ,in Reparatur“ ist (Elkins et al. 2009). In diesem Sinne machen Verfassungen die ungeschriebene Verfasstheit von Sozialität - auch in ihrem Wandel - sichtbar (Alexander 1998, S. 2f.).

Substantiell bestimmen Verfassungen über die relevanten administrativen Arrangements, über Regierungsform und Gewaltenteilung und über die regelgebenden Verfahren. Sie bestimmen aber auch darüber, wer oder was durch die Verfassung geordnet werden soll: Bürger*innen, korporative Akteure, Verwaltungseinheiten. Darüber hinaus haben sie Konsequenzen für das Verhältnis der Bürger*innen untereinander und zum Staat: Verfassungen bestimmen die entscheidenden Rechte und Pflichten, die Bürger*innen vor dem staatlichen Zugriff schützen sollen. Die damit einhergehende einschränkende Reglementierung staatlicher Machtverhältnisse (u. a. Waluchow 2018) soll hier jedoch weniger im Vordergrund stehen als die durch Rechte und Pflichten formulierten Verständnisse dessen, was als good citizenship gelten soll.

Aus diesem Moment der substantiellen Dimension speist sich auch die deklarative Dimension der Verfassungen als ,performativer Akt“: ${ }^{4}$ Verfassungen manifestieren das kollektive nationale Selbstverständnis und bestimmen das „wir“ des gemeinschaftlichen Demos der staatlichen Entität, ${ }^{5}$ indem sie ihre Mitglieder benennen und dadurch bestimmen und vereinigen: ,,[... in nations with heterogeneous populations [...] a constitution can serve as an expression, perhaps the only one available, of national unity“ (Tushnet 1983, S. 1984). Damit formatieren Verfassungstexte sowohl individuelle als auch kollektive Einheiten. Nicht zuletzt diese deklarative Dimension von Verfassungen gibt immer wieder Anlass für Überarbeitungen, Erweiterungen und Neuverhandlungen von Verfassungstexten (Thornhill 2017, S. 495).

\subsection{Verfassungen als Identitätsangebote}

Soziologische Beiträge zu Verfassungen und ihrem ordnenden Charakter entwerfen eine vielfältige Perspektive auf ihre gesellschaftliche Verortung: Sie befassen sich mit unterschiedlichen Verfassungskulturen (z. B. Häberle 1998; Witte und Bucholc 2016), mit der historischen Einbettung und den gesellschaftlichen Konstellationen ihrer Entstehung (Boli-Bennett 1979; Go 2003; Elkins et al. 2009; Thornhill 2011; Schmidt 2012), mit Fragen nach der Integrationsfähigkeit von Verfassungen (Vorländer 2002), mit Verfassungen als symbolischer Ordnung (Vorländer 2017) oder mit dem Spannungsverhältnis von nationalen Verfassungen und Globalisierung (Boli-

\footnotetext{
4 Das Konzept des performativen Aktes als Akt der Hervorbringung dessen, was er benennt, wird hier in Anlehnung an Butler (u. a. 1988) verwendet.

5 ,Ethnos“ und „Demos“ sind Begriffe, die es ermöglichen, zwischen Abstammungsgemeinschaften (,,ethnoi“) und Gemeinwesen zu unterscheiden, die auf gesetzesförmigen Regeln fußen (,demoi“). Dabei geht es zunächst um die Unterscheidung der Art des Gemeinwesens, noch nicht um die Art der Identifikation wie bei den Konzepten der „,civic“ und ,ethnic national identity“ (Jones und Smith 2001).
} 
Bennett 1979; Go 2003; Heintz und Schnabel 2006; Thornhill 2017). Selten werden Verfassungen soziologisch hinsichtlich ihrer identitätsstiftenden Kraft in den Blick genommen. Die folgende Analyse konzentriert sich daher auf den bereits verabschiedeten Verfassungstext und die darin eingebetteten Identitätsangebote. ${ }^{6}$

Diese Angebote entstehen, wenn Verfassungen in ihrer deklarativen Funktion diejenigen Einheiten festlegen, für die die in ihnen verbriefte Souveränität und Autonomie gelten soll. Verfassungen konstituieren diese Entitäten und deren Einheit - den Demos - indem sie sie benennen und durch Rechte und Pflichten näher bestimmen. Der Demos wird dabei als souverän und autonom definiert und einem Territorium zugeordnet; seine Mitglieder sind Träger*innen von Rechten; sie sind aber auch durch Verpflichtungen an das Gemeinwesen gebunden und werden dadurch erst als Mitglieder bestimmt (Tushnet 1983, S. 1985 ff..). ${ }^{7}$ Damit sind Verfassungen Dokumente, die durch Anrufung verbindliche Identitätsangebote an diejenigen offerieren, die sie als staatliche Einheiten benennen. ${ }^{8}$ Im Gegensatz $\mathrm{zu}$ anderen sozialen und kollektiven Identitäten findet dieser Zusammenschluss nicht realiter statt, ${ }^{9}$ der Verfassungstext selbst bleibt unvollzogen und wird nur mittelbar in Praktiken realisiert so „machen“ Verfassungen Menschen nicht zu Staatsbürger*innen (hierzu werden weitere (Verwaltungs-)Akte notwendig), da Verfassungen selbst keine explizit formulierten Sanktionen umfassen. Die Verbindlichkeit ihrer Identitätsangebote muss verfassungsextern, beispielsweise durch Staatsbürgerschaftstests oder verfassungsschützende Institutionen, geregelt und sanktioniert und durch symbolische Politiken wie Nationalfeiertage, Paraden oder Gedenkstätten abgesichert werden. Damit bleiben Verfassungen Identitätsangebote, die als Bezugspunkt für individuelle und kollektive Identitäten dienen können; diese Identitäten müssen aber erst in der Verfassungswirklichkeit - durch diskursive und institutionelle Dispositive abgesichert realisiert werden.

Die Verfassungstheorie verortet Verfassungsidentitäten auf drei Ebenen (u. a. Jacobsohn 2006; Rosenfeld 2009, 2012; Tushnet 2010): ${ }^{10}$ (i) Allein das Vorliegen einer Verfassung verweist bereits auf eine bestimmte Identität des Gemeinwesens; (ii) die in der Verfassung niedergelegten politischen Gestaltungsprinzipien schaffen je unterschiedliche Demos-bezogene Identitäten; (iii) die verfassungsmäßig verfassten fundamentalen Rechte und Pflichten der Mitglieder konzipieren das Gemeinwesen des

\footnotetext{
${ }^{6}$ Die den Konstitutionalismus im engeren Sinne leitende Frage nach der Limitierung gouvernementaler Macht (Waluchow 2018) wird in diesem Beitrag damit nur implizit adressiert.

7 Für die weiteren Ausführungen wird hier von der formal-rechtlichen Logik der Staatsbürgerschaft abstrahiert und generell von Bürger*innen gesprochen. Diese können sowohl Staatsbürger*innen im formallogischen Sinne umfassen, aber auch noch nicht naturalisierte Migrant*innen oder Asylbewerber*innen. Wenn diese Unterscheidung relevant ist, wird gesondert darauf hingewiesen.

8 Eine vollständige Nicht-Identifikation findet entweder im Rahmen von als verfassungsfeindlich gekennzeichneten Aktivitäten statt oder durch Emigration.

9 Zur Notwendigkeit praktischer Realisierung von sozialen und kollektiven Identitäten vgl. beispielsweise Garfinkel (1967, S. 116ff.) oder Lamont (2001).

10 Rosenfeld (2012, S. 756-757) schreibt hierzu: „Placed in their contemporary setting, conceptions of constitutional identity range from focus on the actual features and provisions of a constitution [...] to the relation between the constitution and the culture in which it operates, and to the relation between the identity of the constitution and other relevant identities, such as national, religious, or ideological identity“.
} 
Demos und identifizieren seine Mitglieder. Diese Bestimmung wird im Folgenden im Vordergrund stehen, adressiert sie doch die im Verfassungstext entworfene Figur des good citizen. Aus dieser Figur wiederum speisen sich sowohl das individuelle Identitätsangebot als auch die daraus abgeleitete ,imagined community“ (Anderson 1988, S. 17), die durch ihren Bezug zu einem juristischen Regelwerk zusammengeschlossen wird: Sie kann als Verfassungs- oder Staatsgemeinschaft mit einer nationenbezogenen Abstammungsgemeinschaft übereinstimmen, muss dies aber nicht (wie z. B. in Belgien, Großbritannien oder Spanien). ${ }^{11} \mathrm{Ob}$ und inwieweit Verfassungen tatsächlich zu integrieren vermögen, ist dabei eine offene Frage (vgl. Vorländer 2002, S. $11 \mathrm{f}$.), die hier jedoch nicht adressiert werden soll.

Das Konzept des good citizen ist der politischen Philosophie entlehnt und wird hier in das Feld der Verfassungen verschoben: Im Rahmen der politischen Philosophie adressieren Debatten um good citizenship, welche Rechte und Verpflichtungen Bürger*innen idealiter in einer Demokratie haben sollten. Dies gehe über gleiche politische Rechte hinaus und umfasse (je nach politischer Richtung) auch unterschiedliche implizite „Tugenden“ wie Gemeinwohlorientierung oder Eigenverantwortung (Kymlicka und Norman 1994). Pykett et al. (2010, S. 526) argumentieren ähnlich, dass die Figur des good citizen immer kontext- und ideologie-abhängig inszeniert werde und damit auch immer normativ aufgeladen sei. Rechtliche Zuweisung von Staatsbürgerschaft und ihr enactment als Bürger*in ließe sich zwar nicht praktisch, aber analytisch trennen. Im Rahmen dieses Beitrags sollen Verfassungen als ein Kontext gelten, in dem nicht nur Staatsbürgerschaft verhandelt wird (vgl. hierzu: Behrens 2021), sondern auch Fabrikation und Inszenierung des good citizen jenseits einer rechtlich verfassten Staatsbürgerschaft erfolgt. Damit wird die Figur des good citizen nicht nur für demokratische Regierungsformen relevant, sondern in allen Verfassungen aufspürbar. Good citizenship ist damit einerseits Ausdruck von dem, was sich als Verfassungskultur zusammenfassen lässt, ${ }^{12}$ andererseits verfassungsförmig idealisierter Bezugspunkt für kollektive und individuelle Identitäten, die in der alltagsweltlichen Verfassungswirklichkeit je unterschiedlich realisiert werden. Die folgende Analyse wendet die Frage, wie sich good citizenship in Verfassungen ausdrückt, empirisch und untersucht sie im Feld der Religion.

\section{Der Datenkorpus des Religion and Nation in Constitution worldwide Dataset (RNCw)}

Um die Identitätsangebote von Verfassungen genauer untersuchen zu können, verwenden wir Daten, die auf der Basis einer explorativen Dokumentenanalyse von Verfassungstexten akquiriert wurden. Die Daten wurden im Rahmen des DFG-ge-

\footnotetext{
${ }^{11}$ Hinzu kommt, dass das verfassungsförmige Identitätsangebot immer in seinen Entstehungskontext eingebettet ist: Dies kann zu Konflikten führen, wenn sich der Demos durch technischen, ökonomischen, sozialen Wandel oder durch $\mathrm{Zu}$ - und Abwanderung ändert, der Verfassungstext aber unverändert bleibt (Tushnet 2010, S. 676).

12 Unter dem Begriff der Verfassungskultur lässt sich zusammenfassen, dass und inwieweit Verfassungsnormen eine kulturelle Basis haben und im kulturellen Kontext, in dem sie entstanden sind, verankert sind (Vorländer 2002; Schmidt 2012).
} 
förderten Projekts ONBound zusammengestellt. ${ }^{13}$ Dabei ging es darum, Verfassungen daraufhin zu untersuchen, inwiefern sie Religion und Religiosität (aber auch Nation und Nationalität, die hier aber nicht weiter analysiert werden) arrangieren und reglementieren.

Die soziologische Relevanz von Verfassungstexten liegt nicht zuletzt darin, dass in diesen Texten Recht und Gesellschaft in ein grundlegendes Verhältnis gestellt und dieses Verhältnis dokumentiert wird (Heintz und Schnabel 2006, S. 692f.). Witte und Bucholc (2016, S. 4) weisen darüber hinaus darauf hin, dass Verfassungstexte sozial hochrelevante diskursive Praktiken aufrufen: so wird sich auf den Text berufen, er wird als Gründungsdokument ausgestellt und besichtigt, er wird ausgelegt, es wird gegen ihn Beschwerde einlegt, auf ihn geschworen, darum gestritten. Der besondere Vorteil des hier genutzten Datensatzes besteht darin, dass er einen weltweiten Vergleich von Verfassungstexten jenseits spezifischer Falluntersuchungen zulässt; sein Nachteil ist jedoch, dass er die spezifische gelebte Verfassungswirklichkeit der Entstehung und Umsetzung nicht erfasst.

Der Datenkorpus des RNCw entstand wie folgt: ${ }^{14}$ Unter Rückgriff auf die Datenbank des Comparative Constitutions Project „Constitute“ wurden englischsprachige Verfassungstexte erfasst. Zunächst wurde ein Codebuch erstellt, welches alle Dimensionen und dazugehörige Variablen und deren Ausprägungen umfasst, die mit Religion (ihren Synonymen und Wortfeldern) in Verbindung gebracht werden konnten. Das Themenfeld „Religion“ spannt sich entlang der Dimensionen „Staats-Religions-Verhältnis“, „Antidiskriminierung“, „Familie und Schule“, „Religionsfreiheit“, „Regelung religiöser Organisationen“ und „Religion und Verwaltung“ auf.

Das Kodebuch diente als Basis für den eigentlichen Kodierungsprozess. Alle Verfassungen wurden von Kodierer*innen unabhängig voneinander gelesen und dem Codebuch entsprechend verkodet, um Intercoder-Reliabilität herzustellen. Die Ergebnisse dieser Verkodungen wurden regelmäßig gemeinsam abgeglichen. In Zweifelsfällen wurden die entsprechenden Artikel gemeinsam gesichtet und diskursiv eine Einigung über den angemessenen Code erzielt. Der fertige Datensatz umfasst die im Jahr 2017 gültigen Texte von 186 Verfassungen. Dies entspricht einem Länderanteil von $94 \%$ der weltweit 198 durch die UN anerkannten Staaten. ${ }^{15}$ Insgesamt decken 56 Variablen das Themenfeld Religion ab.

Verkodet wurden allein Verfassungstexte, die als einheitliches Dokument zur Verfügung standen. Um die Vergleichbarkeit der Dokumente zu gewährleisten, wurden keine anderen Rechtsdokumente in die Untersuchung einbezogen. Die Verkodung berücksichtigt ausschließlich den verfassungstextlichen Korpus der Verfassungen; um eine weltweite Vergleichbarkeit zu ermöglichen, wurden verfassungstheoretische

13 DFG Projekt Nummer: 316798296 [https://www.onbound.international/Home.aspx], die Daten sind zugänglich unter https://doi.org/10.7802/1991.

14 Vgl. für ein Subset: Schnabel et al. (2017), methodisch ähnlich: Heintz und Schnabel (2006).

15 Die Verfassungen und verfassungsrelevanten Dokumente von Kanada, Israel, Kosovo, Neuseeland, Puerto Rico, San Marino, Saudi-Arabien, Taiwan, Nord- und Süd-Sudan sowie Großbritannien wurden nicht aufgenommen, da diese Staaten über kein einheitliches Verfassungsdokument verfügen oder nicht von der UN anerkannt sind und damit die Vergleichbarkeit der Dokumente nicht hergestellt werden kann. Die Verfassung des Kongo wurde nicht aufgenommen, da sie nicht in englischer Übersetzung auf Constitute, sondern nur im französischen Original verfügbar war. 
Auslegungen und Interpretationen für die Verkodung ebenfalls nicht berücksichtigt. Die folgende Analyse der Verfassungen wiederum folgt einer soziologischen Logik, verfassungsrechtliche Argumente werden nur im Sinne dieser Logik aufgenommen.

Der RNCw unterscheidet sich von anderen verfassungsvergleichenden Projekten wie dem Religion And State Constitutions Dataset (RAS, Fox 2014) und dem Comparative Constitutions Project (CCP, Elkins et al. 2014) und stellt einen genuin eigenständigen Datensatz dar: Während das RAS Constitutions Dataset Verfassungen nur bis 2008 berücksichtigt, bezieht der RNCw Verfassungen ein, die im Jahr 2017 Gültigkeit hatten. Dreißig der von uns verkodeten Verfassungen durchliefen in der Zwischenzeit teilweise umfassende Änderungen. Das CCP wiederum umfasst nur sieben religionsbezogene Dimensionen zum Staat-Religionsverhältnis und legt seinen Schwerpunkt eher auf Entstehungs- und Veränderungskontexte, während der RNCw-Datensatz genuin verfassungstextbasiert ist und dreizehn Dimensionen adressiert, die neben dem Staat-Religionsverhältnis auch Regelungen zu religiösen Organisationen, zu Religion und Schule sowie zur Religiosität von Staatsdiener*innen berücksichtigt. ${ }^{16}$

\section{Präambeln und ihre Identitätsangebote}

Präambeln kommt eine besondere Rolle innerhalb der Verfassungstexte zu: Sie sind gleichzeitig Einleitung und Deklaration und werden durch ihren spezifischen Inhalt bestimmt (Kelsen 1946, S. 134). ${ }^{17}$ Präambeln lassen sich als den Teil der Verfassung lesen, in dem Identitätsangebote in besonderer Weise textlich verfasst werden: In ihnen wird der verfassungstextliche Bedeutungsüberschuss besonders sichtbar (z. B. Häberle 1982).

\subsection{Der Stellenwert der Präambel}

In der Rechtsliteratur werden der Präambel politisch-ideologische und symbolische Funktionen zugeordnet (Kelsen 1946, S. 143; in Anlehnung an Kelsen: Orgad 2010, S. 738), die insbesondere identitätsformatierend relevant werden:

Politisch-ideologisch zeigt das Vorliegen von Präambeln an, dass es gelungen ist, eine Übereinkunft auch jenseits bestehender gesellschaftlicher Spaltungen über ein verbindlich gültiges, grundlegendes Regelwerk herzustellen. Präambeln behaupten dabei, dass die Sozialität, der dies gelungen ist, souverän sei und die Legitimation habe, eine solche Einigung herzustellen (Kelsen 1946, S. 140). Nicht zuletzt diese in der Präambel niedergelegte, textlich verfasste Übereinkunft lässt sich als basales Moment der Interpellation des kollektiven Verfassungssubjekts (etwa auf der ersten Ebene, vgl. Abschnitt 2.2) lesen.

\footnotetext{
16 Das Codebook zum RNCw-Datensatz kann eingesehen werden unter: https://search.gesis.org/research_ data/SDN-10.7802-1991. Ein systematischer Vergleich der Verkodungen zwischen RAS Constitutions Data, CCP Data und RNWc-Daten steht noch aus.

17 Ihre rechtlich bindende Funktion und ihre interpretative Funktion sind hier nicht von Belang.
} 
Das kollektive Verfassungssubjekt wird durch die symbolische Funktion von Präambeln weiter bestimmt, indem Präambeln das ,wir“ der Sozialität in vielfacher Weise beschreiben (Orgad 2010, S. 738 sowie Addis 2018, S. 128). So umfassen Präambeln oft Entstehungsnarrative, die besondere, als erinnerungswürdig angesehene Ereignisse hervorheben (z. B. die Präambel der ägyptischen oder japanische Verfassung); sie benennen Werte und Ziele, die die Sozialität verbindlich prägen sollen (,celebrating Mother Earth“ (Ecuador), ,recognise the equal worth of men and women“ (Zambia) oder ,,reaffirm our determination to build a Rule of Law in which human rights, public freedoms, human dignity, justice and good governance [...] are promoted“" (Elfenbeinküste)) und sie beschreiben Sozialitäten als säkular oder einem Gott verpflichtet (vgl. 4.2). Damit haben Präambeln einende Funktionen und können über gesellschaftliche Risse hinweg Gemeinschaft proklamieren. Für Addis (2018, S. 137 ff.) haben Präambeln ,biographischen“ Charakter, analog zu individuellen Autobiographien. Diese Vorstellung impliziert, dass Präambeln ein unterscheidbares Subjekt konstituieren, das sich durch Verweise auf gemeinsame Geschichte, Ziele und Überzeugungen charakterisieren lässt. Das solchermaßen fabrizierte Kollektivsubjekt lässt sich als Identitätsangebot für aktuelle, aber auch zukünftige Mitglieder des Kollektivs lesen. Dabei können Präambeln genauso vereinigende wie auch exkludierende Wirkung entfalten: So stieß etwa im Fall von Mazedonien die Formulierung ,,,national state of the Macedonian people“ as well as other nationalities that reside therein" auf Protest der albanischen Bevölkerung, die sich dadurch zur Minderheit degradiert sah (Orgad 2010, S. $731 \mathrm{ff}$.).

Im Folgenden wird gezeigt, welche Identifikationsangebote Präambeln in ihrer ideologisch-politischen und symbolischen Funktion speziell in Bezug auf religiöse Identitäten machen.

\subsection{Identitätsangebote innerhalb der Präambeln}

Von den 186 von uns verkodeten Verfassungen verfügt die überwiegende Mehrheit von 156 Verfassungen über eine Präambel. Im Sinne einer weltgesellschaftlichen Perspektive lässt sich hier von einem formalen Isomorphismus sprechen (BoliBennett 1979; Blaustein 1994). Differenzen zeigen sich aber, wenn Religion als wichtiges Moment der in den Präambeln aktivierten symbolischen Gemeinschaften (Orgad 2010, S. 717) in den Blick genommen wird: Wie Abb. 1 zeigt, findet sich zwar in mehr als der Hälfte der Präambeln ein Gottes- oder Religionsbezug (87; $55 \%$ ), jedoch in unterschiedlicher Tiefe. In einigen Präambeln stellt der Gottesbezug eine besondere Autorisierung der folgenden Rechtsnormen dar. So beginnt z. B. die Schweizerischen Bundesverfassung mit „Im Namen Gottes des Allmächtigen!“ In anderen Präambeln erfolgt aus dem Gottes- oder Religionsbezug eine besondere Verantwortung, wie z.B. in der Formulierung, die im deutschen Grundgesetz zu finden ist: „Im Bewußtsein seiner Verantwortung vor Gott und den Menschen“. In wieder anderen Präambeln wird für die Realisierung der Verfassung auf Gott vertraut oder dessen Hilfe in Anspruch genommen. ${ }^{18}$ Ein konkreter Gott oder eine konkrete

18 So heißt es in der Präambel zur Verfassung von Bahrain: „In the name of God on high, and with His Blessing, and with His help“. 
explizite Religionsnennung

generelle Nennung

Säkularität

keine Nennung

keine Präambel
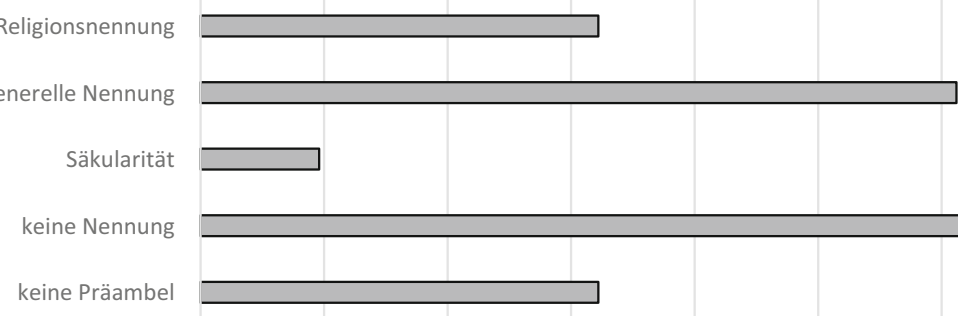

0

5

10

15

20

25

30

35

Abb. 1 Religionsbezug in Präambeln (prozentualer Anteil an allen Verfassungen mit einer Präambel in \%). (Quelle: RNCw - Eigene Berechnungen)

Religion werden in 30 (19,8\%) Präambeln benannt. Dabei wird entweder das Land als ein einer spezifischen Religion verpflichtetes beschrieben (z.B. in den Präambeln der Verfassungen von Algerien oder Thailand), der Demos wird konfessionell zugeordnet (z.B. in der Präambel der Verfassung von Nicaragua), oder die Präambel benennt einen spezifischen Gott (z. B. Iran). In den 30 Präambeln, in denen der Gottes- oder Religionsbezug konkretisiert wird, wird in 12 Präambeln auf den Islam oder Allah rekurriert, in 15 auf das Christentum und in drei Fällen auf Buddha. Säkularität wiederum ist nur in 9 Präambeln $(5,7 \%)$ verpflichtend festgeschrieben. ${ }^{19}$

Der Gottes- oder Religionsbezug in der Präambel verlangt jedoch nicht, dass die individuellen Mitglieder des Demos selbst religiös sein müssten. Die Aktivierung des Göttlichen oder Religiösen in der Präambel markiert den Stellenwert für die Selbstbeschreibung des Kollektivs und konstituiert damit ein weltanschaulich besonders verfasstes Gemeinwesen. Damit geht ein besonderes Identitätsangebot an die Mitglieder einher, sich diesem weltanschaulichen verfassten Kollektiv anzuschließen und zu verpflichten, mit einer entsprechenden Macht zur (symbolischen wie faktischen) Exklusion derjenigen Minderheiten, die diesem Kollektiv nicht angehören.

Viele Präambeln, die einen Gottes- oder Religionsbezug beinhalten, sind im Namen eines verfassungsgebenden Demos verfasst: Mit der Formulierung „We, the people ..." wird der Demos, den die Verfassung erst begründen soll, bereits als verfassungsgebende, souveräne, stabile und vereinte Kraft jenseits möglicher Konfliktlinien anerkannt und aufgerufen, selbst wenn die Verfassung nicht in einer „Urabstimmung“ verabschiedet wurde. ${ }^{20}$ Der Zusammenhang zwischen Gottes- oder Religionsbezug und der Aktivierung eines solchermaßen kollektivierenden ,wir““ ist signifikant und deutlich (Phi $=0,713, p<0,001)$. Insgesamt 36 Präambeln haben beides:21 Hier findet sich die stärkste symbolische Verpflichtung des Demos als religiöse Gemeinschaft, diese Präambeln machen damit ein religionsbezogenes

\footnotetext{
19 Dies war zum Zeitpunkt der Verkodung für die Verfassungen der folgenden Staaten der Fall: Aserbaidschan, Bangladesch, Kamerun, Elfenbeinküste, Indien, Mali, Namibia, Türkei und Turkmenistan.

20 In diesen Fällen spricht Addis (2018, S. 138 [Hervorhebung nicht im Original]) davon, dass ,,[o]wnership and authorship are declared clearly and unambiguously.".

21 Eine entsprechende Kreuztabelle findet sich im Anhang (Tab. 3).
} 
Identitätsangebot an die aufgerufene Gemeinschaft. Exemplarisch findet sich dies z. B. in der Präambel zur irischen Verfassung, in der der Autorenschaft des ,We, the people“ die Verpflichtung auf die christliche Dreieinigkeit vorangestellt wird. ${ }^{22}$

Darüber hinaus weisen Präambeln von Verfassungen de-kolonialisierter Länder des globalen Südens eher einen Gottes- oder Religionsbezug auf (Pearsons r: 0,206, $p=0,01)^{23}$; Länder des ehemaligen Ostblocks eher seltener (Pearsons r: $-0,244$, $p=0,002)$. Ersteres könnte darauf zurückzuführen sein, dass Religion im Prozess der De-Kolonialisierung für viele Länder des globalen Südens ein kollektives Abgrenzungs- und Identitätsmerkmal ist. Letzteres hingegen lässt sich als Nachhall der gouvernementalen Religionsferne des ehemaligen Sowjet-Regimes lesen.

Die Kehrseite der Fabrikation eines durch die Präambel religiös vergemeinschafteten Demos ist, dass Diversität und Wandel zu Exklusion, Kritik und Distanz führen können: So werden a-religiöse Menschen oder religiöse Minderheiten allein auf der Basis des Wortlauts von Präambeln exkludiert, wenn der Gottes- oder Religionsbezug keine Nennung von Minderheiten oder andere Inklusionsangebote umfasst (Addis 2018, S. 169; Orgad 2010, S. 730). Empirisch beinhalten von den 87 Präambeln mit einem Gottes- oder Religionsbezug nur sechs Präambeln die Anerkennung ethnischer Minderheiten und 15 Präambeln die Anerkennung von Minderheiten ohne Spezifikation; allein die Präambel zur Verfassung von Burundi erkennt explizit religiöse Minderheiten an. In den anderen Fällen werden Minderheiten aber auch Immigrant*innen und zukünftige Generationen auf ein Identitätsangebot festgelegt, in dem sie nicht vorkommen; sie sind damit nicht unmittelbare Adressat*innen dieses Identitätsangebots, dessen Verpflichtungscharakter - so lässt sich vermuten geschwächt wird.

\section{Religionsfreiheit und ihre Einschränkung}

Während die Präambeln in unterschiedlichem Maße kollektive Identitäten schaffen, konstituieren die Verfassungstexte selbst die Mitglieder des Demos durch die Formulierung bestimmter individueller Rechte und Pflichten (Böckenförde 2011, S. 13 ff.). In diesen Rechten und Pflichten manifestiert sich ein bestimmtes - verfassungsspezifisches - Verständnis des*der Staatsbürger*innen und es wird bestimmt, was einen (idealtypischen) good citizen ausmacht.

\subsection{Religionsfreiheit als Freiheits-Paradox}

Religionsfreiheit als Komplex individueller Rechte nimmt in den meisten Verfassungen einen besonderen Stellenwert ein, nicht zuletzt deswegen, weil sich das Recht auf individuelle Religionsfreiheit historisch aus der Konkurrenz zwischen der neu

\footnotetext{
${ }^{22} \mathrm{Zu}$ Begin der Präambel heißt es: „In the Name of the Most Holy Trinity, from Whom is all authority and to Whom, as our final end, all actions both of men and States must be referred". Aber auch hier wird Religiosität nicht individuell eingefordert, sondern das nationale Kollektiv als religiös charakterisiert und dem (katholischen) Glauben verpflichtet.

23 Die Variable „Gottesbezug in Präambel“ wurde für diese Berechnung dichotomisiert.
} 
entstehenden Staatssouveränität und den Machtansprüchen religiöser Gruppierungen entwickelt hat (Sajó und Uitz 2012, S. 909). Dies bedingt sowohl die Vielfältigkeit der Formen, in denen Religion in Verfassungen verankert ist, als auch die Sonderstellung, die die verfassungsmäßige Garantie der Religionsfreiheit unter anderen individuellen Freiheiten einnimmt (etwa Bock 1998, S. 444).

Im Folgenden soll jedoch weniger die individuelle Freiheit von und zur Religion sowie ihre gemeinschaftliche Bekundung und Ausübung als schützenswerter Tatbestand im Vordergrund stehen, ${ }^{24}$ als vielmehr die Frage, wie Verfassungen ihre Bürger*innen als ,religiöse“ und ,nicht-religiöse“ Subjekte verfassen.

Religionsfreiheit wird im (rechts-)theoretischen Diskurs im Kontext der individuellen Gewissensfreiheit verortet. Religionsfreiheit gründet auf der Vorstellung, religiöse Überzeugungen müssten als Ergebnis einer fundamentalen individuellen Entscheidung angesehen werden: ,[B]eliefs are central for the individual, and respect for the individual entails respect of his personal choices" (Sajó und Uitz 2012, S. 914; ähnlich: Bock 1998, S. 449 ff.). Hier spiegelt sich die der westlichen Moderne eingeschriebene Werthaltung, die das Individuum als autonome Entität mit eigenem Willen, individuellen Wünschen und Bedürfnissen entdeckt (van Dülmen 2016). Damit werden Staatsbürger*innen konzipiert, die in ihrer ,,inneren“ Freiheit zu glauben und ihrer ,äußeren“ Freiheit, diesen Glauben auch zu manifestieren, vor dem Zugriff des Staats und anderer Mitbürger*innen geschützt werden müssten (vgl. u. a. Bock 1998, S. 446). Dies umfasst auch die Freiheit, nicht zu glauben oder einem Glauben anzuhängen, der den religiösen Überzeugungen der Mehrheit der Staatsbürger*innen nicht entspricht.

Diese individuellen Freiheitsrechte, zu glauben, nicht zu glauben und seinen Glauben mit anderen zu manifestieren, müssen jedoch im Zusammenhang mit dem in den Verfassungen geregelten Staat-Religions-Verhältnis gelesen werden: Es bestimmt darüber, wie umfangreich diese Freiheiten geschützt und realisiert werden können. Dem Staat wird dabei die Aufgabe zugewiesen, sowohl individuelle Freiheiten zu gewähren als auch Frieden zu stiften und Ordnung zu garantieren (Dreier 2018, S. 11). Das westlich-moderne Staat-Religions-Verhältnis fußt verfassungstheoretisch auf drei Grundprinzipien: ${ }^{25} \mathrm{Neben}$ dem staatlich garantierten Diskriminierungsverbot sind dies die institutionelle Nichtidentifikation, also das allgemeine Einmischungsund Interventionsverbot in kirchliche Angelegenheiten und die sachliche Nichtidentifikation, nach der niemand zu einer religiösen Handlung oder Überzeugung gezwungen werden darf (Dreier 2018, S. 98 ff.). Soziologisch gesehen wird also die Macht des Politischen durch das Recht auf Religionsfreiheit eingehegt (Lindemann 2009, S. 110f.).

Hierzu gehört außerdem, dass der Staat seine Mitglieder darauf verpflichtet, die individuellen Glaubensüberzeugungen und Bekundungen auch von Minderheiten zu tolerieren und den eigenen Glauben oder Nicht-Glauben im Rahmen der verfass-

\footnotetext{
24 Hierzu gibt es bereits eine umfängliche Debatte (Böckenförde 1989; Bock 1998; Greenawalt 2008; Grimm 2008; Sajó und Uitz 2012; Dreier 2018).

25 Diese Grundprinzipien werden im Verfassungsrechtsdiskurs vor allem Staatsformen zugeschrieben, die sich als säkulare Verfassungsstaaten (im westlichen Sinne) beschreiben lassen (vgl. Böckenförde 1989; Dreier 2018).
} 
ten gesellschaftlichen Grundordnung zu realisieren. Diese Verpflichtungen, die im Rahmen der Ordnungsfunktion des Staates umgesetzt werden, erlauben und erfordern unter Umständen die Einschränkung individueller Freiheiten. Hier wird das sichtbar, was Benhabib (2010, S. 454) in Anlehnung an (und Abgrenzung zu) Carl Schmitt den ,state of exception“ nennt: ${ }^{26}$ Zur Gefahrenabwehr kann der souveräne Staat Autonomie und Freiheitsrechte seiner Bürger*innen beschneiden und Ausnahmen von der Regel proklamieren. ${ }^{27}$ An der Schnittstelle zwischen der Ausübung staatlicher Souveränität und der rechtlich garantierten Autonomie der Bürger*innen entstehen die Paradoxien der Religionsfreiheit (vgl. Beyer 2018, S. 245 f.): Nicht nur kann individuelle Religionsfreiheit mit anderen individuellen Rechten, die der Staat verfassungstextlich sicherzustellen verpflichtet ist, in Konflikt geraten (z.B. dem Recht auf körperliche Unversehrtheit, wenn religiöse Riten körperliche Eingriffe vorsehen). Darüber hinaus können auch staatlich zu schützende religiöse Kollektive zur Bedrohung der individuellen Freiheiten werden, die es zu vermeiden gilt (z. B. wenn Fundamentalismen gegenseitigem Respekt entgegenstehen oder die Freiheiten der Mitglieder dieser Kollektive bedrohen). Diese Vermeidung wird dann aber unter Umständen zu einer Benachteiligung bestimmter religiöser Kollektive (Reuter 2007). Der Staat muss hier entscheiden, was er nicht entscheiden kann, nämlich, was als Freiheit von und zur Religion gelten soll, und was eben nicht mehr unter „Religion“ fallen darf (vgl. Dreier 2018, S. 119f. sowie Bock 1998).

Im Folgenden sollen verfassungstextlich Überlegungen abgeleitet werden, wie Verfassungen religiöse und nicht-religiöse Bürger*innen konstituieren, welche Identitätsangebote sie machen und welche Verpflichtungen und Begrenzungen sie den idealtypischen good citizens auferlegen.

\subsection{Verfassungstextliche Religionsfreiheit zwischen individueller Autonomie und staatlicher Souveränität}

Das Staat-Religions-Verhältnis weist nicht in allen Verfassungen vollumfängliche staatliche Säkularität auf; vielmehr ist dieses Verhältnis empirisch vielfältig in den Verfassungen verankert (Tab. 1).

$\mathrm{Zu}$ den 39 Verfassungen, die keine expliziten Regelungen zum Staat-ReligionsVerhältnis enthalten, gehören u. a. die Verfassung von Luxemburg, den Niederlanden, von Bosnien-Herzegowina, Chile, Haiti oder Nordkorea. Das Staat-ReligionsVerhältnis muss hier über andere verfassungstextliche Verankerungen wie z. B. die Regelung des Religionsunterrichts beobachtet werden.

Insgesamt verpflichten 82 Verfassungen den Staat explizit auf Neutralität, indem Staat und Religion getrennt werden und/oder Religionsgemeinschaften keine Privilegien erhalten sollen (Tab. 1). Die Trennung von Staat und Kirche wird in

\footnotetext{
${ }^{26}$ Hier schließt sich die Debatte um das Schmitt'sche Diktum an, nach dem souverän ist, wer über den „Ausnahmezustand“ bestimmen kann (vgl. hierzu kritisch Agamben 2017). Benhabib erweitert dieses Diktum dadurch, dass eben nicht nur der „Ausnahmezustand“ selbst, sondern auch schon die Ausnahmen von der Regel durch den Souverän bestimmt werden können.

27 Diese Einschränkungen treffen oft Minoritäten, Migrant*innen und Asylsuchende in besonderem Maße.
} 
Tab. 1 Verankerung von Staat-Religions-Verhältnissen in Verfassungstexten ${ }^{\mathrm{a}}$

\begin{tabular}{llll}
\hline & Staat-Religions-Verhältnis & $\begin{array}{l}\text { Anzahl der } \\
\text { Verfassungen }\end{array}$ & $\begin{array}{l}\text { Anteil an allen } \\
\text { Verfassungen in \% }\end{array}$ \\
\hline Keine Regelungen & & 39 & 21 \\
Neutralität & Säkularität & 36 & 19,4 \\
& Zurückweisung von Privilegien & 34 & 18,3 \\
& Trennung von Staat und Religion & 65 & 34,9 \\
Religionsspezifisches & Privilegien für Religionsgemeinschaften & 56 & 30,1 \\
Identitätsangebot & Staatsreligion & 36 & 19,4 \\
\hline
\end{tabular}

aMehrfachlistungen möglich

Quelle: RNCw - Eigene Berechnungen

65 Verfassungen formuliert. ${ }^{28}$ In der Mehrheit der Verfassungen von Europa (60\%) und von Zentralamerika und der Karibik (54\%) finden sich Formulierungen, religiösen Gemeinschaften generell keine Privilegien einzuräumen. Länder mit kolonialer Vergangenheit enthalten signifikant seltener solche Formulierungen (Phi $=-0,17$, $p=0,02)$; post-sowjetische Staaten signifikant häufiger ( $\mathrm{Phi}=0,42, p>0,00)$. Verfassungen, die den Staat auf Neutralität festlegen, können als dem europäisch-säkularen Menschenbild der Moderne verpflichtet angesehen werden, aus dem sich damit auch ihr Identitätsangebot speist. Hier wird die Identität eines Kollektivs aus autonomen, vom Staat in ihrer Gewissensentscheidung unbehelligten Individuen angeboten.

Die verfassungstextlich verankerte staatliche Neutralität und Äquidistanz zu den verschiedenen Religions- und Überzeugungsgemeinschaften überlässt es also den Individuen, ihre religiöse oder nicht-religiöse Weltanschauung frei zu wählen, da der Demos hier verfassungstextlich keine Bevölkerungsgruppe bevorzugen oder benachteiligen soll (Dreier 2018, S. 97). ${ }^{29}$ Dies ist anders, wenn Religionsgemeinschaften Privilegien eingeräumt werden oder es eine Staatsreligion gibt; dies offeriert ein Identitätsangebot und hebt genau diese Identität aus anderen Identifikationsmöglichkeiten hervor (vgl. u.a. Schnabel et al. 2020). Formulierungen, die religiösen Gemeinschaften Privilegien wie Steuervergünstigungen einräumen (56 Verfassungen, z. B. Art. 26 (3) in der Verfassung der Philippinen) und/oder eine Staatsreligion vorsehen (50 Verfassungen), sind in insgesamt 74 Verfassungen (40\%) enthalten.

Das Auftreten solcher Bevorzugungen von Religionsgemeinschaften steht im umgekehrten Verhältnis zur post-sowjetischen Vergangenheit eines Landes (zur regionalen Verteilung der Privilegien in Verfassungen vgl. Abb. 2): Verfassungen post-sowjetischer Länder enthalten signifikant seltener Formulierungen, die die Bevorzugung bestimmter Religionsgemeinschaften etablieren. Demgegenüber hat über die Hälfte aller asiatischen Länder Verfassungen, die solche Privilegien einräumen. Hierbei

\footnotetext{
28 So formuliert die japanische Verfassung ,The State and its organs shall refrain from religious education or any other religious activity“ (Art. 20) oder die senegalesische Verfassung „The institutions and the religious communities have the right to develop themselves without hindrance [entrave]. They are disengaged from the protection [tutelle] of the State. They regulate and administer their affairs in an autonomous manner" (Art. 24).
}

29 Ähnlich: Bock (1998, S. 449 ff..) oder Sajó und Uitz (2012, S. 914). 


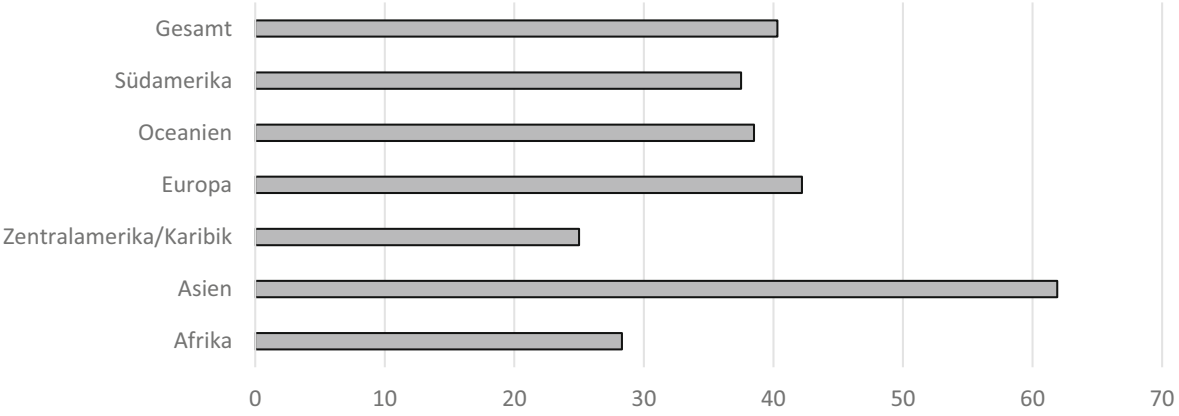

Abb. 2 Verfassungen, die explizit Privilegien für Religionsgemeinschaften/Kirchen beinhalten, nach Region (in \% aller Verfassungen dieser Region). (Nordamerika hat einen Anteil von Null und ist deshalb nicht aufgeführt). (Quelle: RNCw - Eigene Berechnungen)

Zurückweisung von Privilegien UND Trennung von StaatReligion

Gewährung von Privilegien UND Staatsreligion

Trennung von Staat-Religion UND Privilegien für eine Religionsgemeinschaft

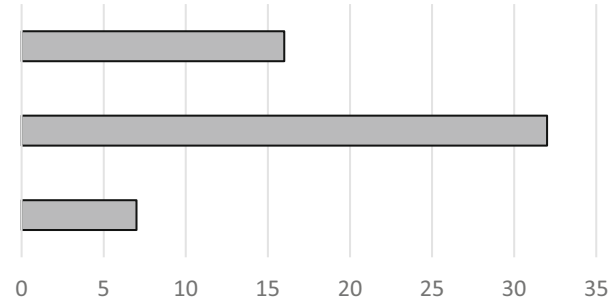

Abb. 3 Anteil der Verfassungen mit Mehrfachnennungen. (Quelle: $\mathrm{RNCw}$ - Eigene Berechnungen)

handelt es sich vor allem um Länder mit muslimischen Majoritätsgesellschaften. ${ }^{30}$ Solche Verfassungstexte legen den Bürger*innen zunächst Religion als weltanschaulichen Bezugspunkt (mehr oder minder verpflichtend) nahe und machen z. T. darüber hinaus noch konfessionell bestimmte Identitätsangebote. ${ }^{31}$ So heißt es etwa in der Verfassung von Bhutan: „Buddhism is the spiritual heritage of Bhutan, which promotes the principles and values of peace, non-violence, compassion and tolerance“ (Art. 3 (1)). Damit werden die good citizens auf eine bestimmte Konfession und auf die damit verbundenen Werte verpflichtet.

Viele Verfassungen enthalten mehrfache Formulierungen zum Staat-ReligionsVerhältnis (Abb. 3).

Jenseits des durch das Staat-Religions-Verhältnis thematisierte kollektive Identitätsangebot ist die individuelle Religionsfreiheit typischerweise in Formulierungen verankert, wie sie sich z.B. in der italienischen Verfassung finden: „Anyone is entitled to freely profess their religious belief in any form, individually or with others, and to promote them and celebrate rites in public or in private" (Art. 19). Damit werden verfassungstextlich Individuen als autonome Entscheidungsinstanzen konstituiert, die über ihr Gewissen eigenständig verfügen können sollen. Nur 9 Verfassungen $(4,8 \%)$ beinhalten keine solchermaßen formulierte individuelle Religionsfreiheit: Es

\footnotetext{
30 So enthalten 16 von $42(38 \%)$ Verfassungen asiatischer Länder einen expliziten Verweis auf die Sharia (afrikanische Länder: 16\%, alle anderen Regionen: 0\%).

31 Der Verpflichtungsgrad ist dann u. a. vom expliziten Schutz von religiösen Minderheiten abhängig.
} 
sind dies die Verfassungen von Algerien, Österreich, Burundi, Komoren, Libyen, Malediven, Mauretanien, Saudi-Arabien und Jemen. Eine explizite Beschränkung dieser Rechte auf Staatsbürger*innen (im Unterschied zu ,,jedermann“) findet sich in 14 Verfassungen.

In 123 Verfassungen (66,1\% aller Verfassungen) werden sowohl die innere Freiheit, diese Überzeugungen privat zu haben, als auch die Möglichkeit, sie mit anderen öffentlich zu manifestieren, garantiert. Nur die Verfassungen des Iran, von Somalia und Tansania beschränken Religionsfreiheit auf private Überzeugungen und Glaubensinhalte ohne Recht auf öffentliche Manifestation. Allerdings verfügen 49 Verfassungen über Formulierungen, die Religionsfreiheit nicht weiter spezifizieren und nicht zwischen ,innerer Überzeugung“ und ,äußerer Manifestation“ unterscheiden. ${ }^{32}$

Wichtiger Bestandteil ist neben der Freiheit zur Religion (positive Religionsfreiheit) auch die Freiheit, keiner (bestimmten) Religionsgemeinschaft angehören zu müssen. Diese negative Religionsfreiheit findet sich in der Garantie, sich nicht einer religiösen Gemeinschaft anschließen oder deren kultische Handlungen ausführen zu müssen, sowie in der Freiheit, eigene religiöse oder weltanschauliche Überzeugungen nicht offenlegen zu müssen. Während immerhin 89 Verfassungen (48\%) solche Formulierungen nicht kennen, findet sich in 62 Verfassungen $(33 \%)$ die Garantie, nicht zu einer (bestimmten) Religion gezwungen werden zu können, 12 Verfassungen $(6,5 \%)$ garantieren, dass Glaubensüberzeugungen nicht öffentlich gemacht werden müssen und 23 Verfassungen $(12,4 \%)$ umfassen beide Formen der negativen Religionsfreiheit. Hier manifestiert sich exemplarisch die westlich-moderne Idee von Bürger*innen als Gleiche unter Gleichen, die nicht zu religiösen Überzeugungen gezwungen werden können. Der Staat verpflichtet sich hier nicht nur selbst, sondern nimmt auch gleichzeitig die good citizens in die Pflicht zu gegenseitigem Respekt (Sajó und Uitz 2012, S. 914).

Eine solche Verpflichtung des good citizen manifestiert sich ebenfalls in dem verfassungstextlichen Verbot, Menschen auf der Basis ihres Glaubens zu diskriminieren oder zu bevorzugen. Auch wenn diese Formulierung zunächst ebenfalls eine Selbstverpflichtung des Staates zur Äquidistanz ist, so spiegelt sie doch darüber hinaus die Forderung an die Bürger*innen, in einem geordneten Gemeinwesen einander Achtung und Respekt entgegenzubringen (Nussbaum 2008, S. 23 ff..). Die Normen der Nicht-Diskriminierung sind fast so verbreitet wie die Manifestation der positiven Religionsfreiheit: In nur 32 Verfassungen (17\%) findet sich weder eine Verankerung von Gleichheitsgeboten noch von Diskriminierungsverboten, die sich auf die Glaubensüberzeugungen beziehen. Alle anderen Verfassungstexte umfassen Gleichheitsund/oder Antidiskriminierungsgebote.

Trotz dieser Rechte und Verpflichtungen birgt Religionsfreiheit aber immer auch die Gefahr, dass religiöse Überzeugungen mit der Verfassungsordnung nicht vereinbar sind (Sajó und Uitz 2012, S. 913): „[Religious behavioral outcomes] have the potential to disrupt the political order as they might lead to disobedience, resistance,

\footnotetext{
32 So findet sich etwa in der Verfassung von Bosnien-Herzegowina die Formulierung: „All persons within the territory of Bosnia and Herzegovina shall enjoy the human rights and fundamental freedoms referred to in paragraph 2 above; these include: [...] g. Freedom of thought, conscience, and religion“ (Art. 3).
} 


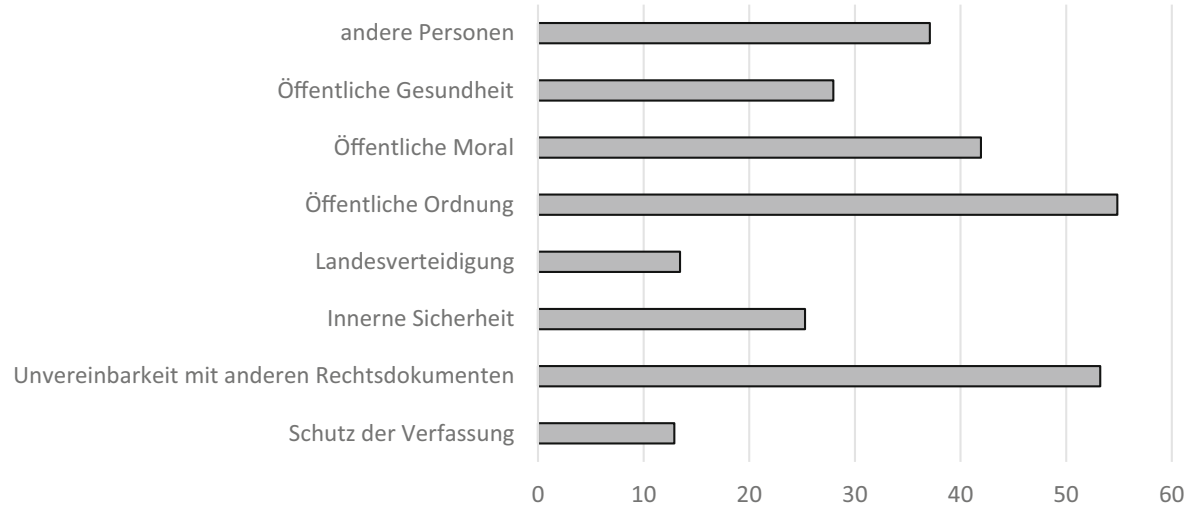

Abb. 4 Intrakonstitutionelle Gründe für die Einschränkung der Religionsfreiheit (in \% aller Verfassungen). (Quelle: RNCw - Eigene Berechnungen)

and martyrdom, which fuel distruptive religious conflicts". ${ }^{33}$ Staaten sehen sich hier in der Pflicht, sowohl ihre rechtliche als auch ihre öffentliche Ordnung - und damit auch ihre Bürger*innen - zu schützen. Viele Verfassungen sehen daher intrakonstitutionelle Klauseln zur Gefahrenabwehr vor. Solche Formulierungen haben typischerweise eine Form, wie sie in der Verfassung von Lesotho zu finden ist: „Nothing contained in or done under the authority of any law shall be held to be inconsistent with or in contravention of this section to the extent that the law inquestion makes provision - a. in the interests of defence, public safety, public order, public morality or public health; or $b$. for the purpose of protecting the rights and freedoms of other persons, including the right to observe and practice any religion without the unsolicited intervention of members of any other religion“ (Art. 13). Solche und ähnliche Formulierungen lassen sich als Indikator staatlicher Souveränitätsbehauptung lesen; sie schränken die individuelle Religionsfreiheit notwendigerweise ein und formatieren den good citizen zu staatstreuen Bürger*innen. Abb. 4 gibt einen Überblick über die in den Verfassungen genannten Gründe. Hierunter findet sich auch wieder die explizite Verpflichtung der Bürger*innen auf gegenseitigen Respekt; diese ist in 69 Verfassungstexten verankert. ${ }^{34}$

Viele Verfassungen nennen jedoch nicht nur einen Grund, sondern bieten oft eine umfängliche Liste: Nur 45 Verfassungen schränken die Religionsfreiheit nicht ein; immerhin 21 Verfassungen $(11,3 \%)$ nennen sieben unterschiedliche Gründe (Abb. 5). Die Anzahl der genannten Einschränkungsgründe ist höher, wenn die erste Landesverfassung relativ jung ist (Peason's R: $0,272, p<0,000$ ). Dies gibt Anlass zu der Vermutung, dass die religiöse Identität von Bürger*innen an Selbstverständlichkeit verliert und Staaten sich zunehmend gegen Anlässe religiöser Störungen abzusichern versuchen.

\footnotetext{
33 Ähnlich argumentiert auch Dreier (2018): Für eine ausführliche Diskussion vgl. Grimm (2008).

34 In der kirgisischen Verfassung findet sich hierzu eine andere Formulierung mit ähnlichem Inhalt: „The propagation of national, ethnic, racial and religious hatred, gender as well as other social supremacy which calls to discrimination, hostility and violence shall be prohibited“(Art. 31).
} 


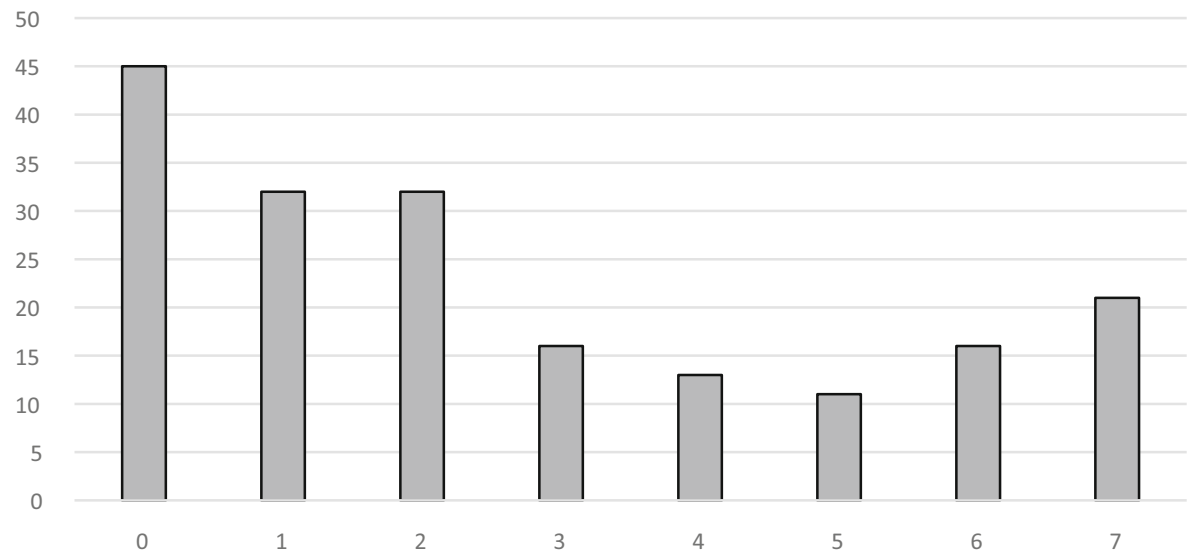

Abb. 5 Häufigkeit der intrakonstitutionell genannten Einschränkungen (in \% aller Verfassungen). (Quelle: $\mathrm{RNCw}$ - Eigene Berechnungen)

Der Blick auf die in den Verfassungstexten verfasste positive und negative Religionsfreiheit legt nahe, dass die weitaus meisten Verfassungen Bürger*innen (im Sinne der Menschenrechte) als Individuen konstituieren, die über ihre religiösen Überzeugungen autonom bestimmen können sollen. Im Rahmen des Staat-Religions-Verhältnisses machen Staaten hier allerdings mit unterschiedlichem Verbindlichkeitsgrad Identitätsangebote: Diese reichen von der verfassungstextlichen Neutralität des Staates (z.B. am prominentesten ist eine solche Neutralität vielleicht in der Verfassung von Frankreich zu finden, aber auch in den Verfassungen von Angola, Ecuador, Indien, Kirgistan, Senegal oder der Türkei) über die Gewährung staatlicher Privilegien für Religionsgemeinschaften (z.B. Afghanistan, Ägypten, Irak, Iran, Island, Italien, Laos, Peru oder Polen) bis hin zu Staatsreligionen, die den good citizens ein bestimmtes Glaubensbekenntnis nahelegen (z. B. Algerien, Argentinien, Finnland, Katar, Malta, Norwegen, Oman, Thailand oder die Vereinigten Arabischen Emirate). Mit der in den Verfassungstexten implizierten Autonomie des Individuums gehen allerdings auch Verpflichtungen des good citizen einher: Diese umfassen sowohl die Verpflichtung auf gegenseitige Toleranz - wie sie sich in der negativen Religionsfreiheit als auch in den Gleichheits- und Anti-Diskriminierungsgeboten spiegeln - als auch die Anerkennung der staatlichen Ordnung. In diesem Zusammenhang legt die Untersuchung der Verfassungstexte nahe, dass viele Staaten ihren (religiösen) Bürger*innen auch ,misstrauen“, wenn sie zur Aufrechterhaltung ihrer Souveränität intrakonstitutionell als „letzte Schranke“ Situationen für mögliche Einschränkung der Religionsfreiheit spezifizieren. ${ }^{35}$ Die Zunahme an Einschränkungsgründen in jüngeren Verfassungen (Pearsons $\mathrm{r}:-0,275, p<0,00$ ) lässt in

\footnotetext{
35 Agamben (2017) argumentiert in seinem Essay, dass diese Möglichkeit der Einschränkung individueller Freiheiten politisch folgenreich für demokratische Systeme sein kann, da mit einer ausnahmsweise ergriffenen provisorischen Maßnahme eine Verschiebung zu einer Technik des Regierens einhergeht, die „,die Struktur und den Sinn der traditionellen Unterscheidung der Verfassungsformen radikal zu verändern [droht]“" (Agamben 2017, S. 9).
} 
diesem Zusammenhang vermuten, dass die religiöse Identität von Bürger*innen an Selbstverständlichkeit verliert und Staaten sich zunehmend gegen Anlässe religiöser Störungen abzusichern versuchen.

\section{Religion, Staat, Erziehung}

Verfassungen enthalten nicht nur Identifikationsangebote für gerade lebende Bürger*innen; sie verfassen auch Aspekte, wie future citizens ,gemacht“ werden sollen. Sie legen damit die Wege fest, auf denen ein zukünftiger Demos an die verfassungstextlichen religionsbezogenen Identitätsangebote herangeführt werden soll. Dies erfolgt prominent über die Bestimmung der Verantwortlichkeiten für die schulische Unterweisung. So finden sich in vielen Verfassungen Regelungen zur Verortung des schulischen Religionsunterrichts, der Autonomie religiöser Unterweisungsinstitutionen oder darüber, ob die religiöse Erziehung in die Verantwortung der Eltern fallen soll oder nicht. ${ }^{36}$ Diese Regelungen werden im Folgenden vor dem Hintergrund der Theorieperspektive der Biopolitik diskutiert.

\subsection{Die Perspektive der Biopolitik}

Foucault definiert Biopolitik als ,techniques of power present at every level of the social body and utilized by very diverse institutions“ (Foucault 1978, S. 141). Neben der Familie gehört für ihn vor allem die Schule zu den zentralen Institutionen, die die Techniken der Überwachung und der Disziplinierung des Körpers bereits in frühester Kindheit übernehmen. Mit der Entwicklung des modernen Staates im Verlauf des 18. Jahrhunderts seien auch diejenigen Machtmechanismen etabliert worden, die Menschen zu Mitgliedern des Staates, mithin zu Staatsbürger*innen, machten. ${ }^{37}$ Für Foucault gehört hierzu auch, dass der Staat mit seinen Machttechniken die Bürger*innen normiert (und normalisiert) ${ }^{38}$ und in ihre Körperlichkeit, Sexualität und Reproduktion eingreift, wobei die Sexualität das Bindeglied zwischen individuellen Körpern und gesellschaftlicher Bevölkerung darstellt. Diese Dimension des Körpers soll hier nicht im Vordergrund stehen, auch wenn sich nicht übersehen lässt, dass Staatsbürgerlichkeit immer an einen solchen Körper gebunden ist, denn auch Recht, das nicht unmittelbar auf Körper zugreift und diese reglementiert, stellt - wenn auch stillschweigend - immer auf die Verbindung von Staatsbürgerlichkeit und Körper $\mathrm{ab}$, welche erst erlischt, wenn der Körper vernichtet ist. In diesem Sinne unter-

\footnotetext{
36 Die verfassungstheoretische Auseinandersetzung mit diesem Themenkomplex ist nicht umfangreich: So finden sich im Oxford Handbook of Comparative Constitutional Law (Rosenfeld und Sajó 2012) weder ein eigenes Kapitel zur verfassungstextlichen Verankerung von Erziehung und Schulunterricht noch entsprechende Einträge im Stichwortverzeichnis.

37 Foucault selbst spricht hier von einer Verschiebung der Macht hin zu einem Souverän, der nicht länger sterben macht und leben lässt, sondern leben macht und sterben lässt (Foucault 1978, S. 138). Im Folgenden soll vor allem das im Vordergrund stehen, was Foucault selbst als Bio-Macht bezeichnet, nämlich die Macht, die sich auf die Regulierung von Bevölkerung bezieht.

38 Robertson (1990, S. 49) beschreibt dies als die Fabrikation von ,standardised citizens“.
} 
werfen staatsbürgerliche Reglementierungen immer den menschlichen Körper den jeweiligen institutionellen Arrangements.

Nicht zuletzt deshalb sind die verfassungstextlichen Regelungen der (religiösen) Unterweisung ein wichtiger Indikator dafür, wie ein Staat in die Sozialisation seiner Mitglieder in Form von körperlicher und geistiger Disziplinierung und Normierung eingreift, diese kontrollieren kann oder sie unangetastet anderen Institutionen überlässt. Verfassungen formulieren also nicht nur Identitätsangebote, sie halten auch - in unterschiedlichem Maße - rechtliche Vorkehrungen bereit, future citizens auf diese Angebote vorzubereiten: Insofern Verfassungen immer vorläufiger Konsens sind, wird über die verfassungsmäßige Reglementierung des staatlichen Zugriffs auf schulische Unterweisung dafür Sorge getragen, dass dieser Konsens und das daran geknüpfte Identitätsangebot an die folgende Generation weitergegeben werden kann.

Das Feld der religiösen Unterweisung hat dabei Indikatorfunktion einerseits für die Neutralität des Staates in Weltanschauungsfragen, andererseits aber auch für die Souveränität des Staates, die Sozialisation der Staatsbürger*innen unabhängig von religiösen Organisationen zu formen und zu bestimmen. Die verfassungstextliche Verankerung und Reglementierung der Instanzen der religiösen Sozialisation ist also insofern von besonderem Interesse, weil es hier eben nicht allein um Identifikationsangebote oder die Verpflichtungen des good citizen geht. Vielmehr manifestieren sich hier die Ideen und Vorstellungen, die die Verfassungsgemeinschaft des Demos darüber hat, welche Institutionen im Dreieck von Staat, Religionsgemeinschaften und Eltern für die religiöse und weltanschauliche Perspektivierung der future citizens Verantwortung übernehmen und wie diese Perspektivierung aussehen soll (Czermak 2008, S. 65 ff.).

Hier geht es nicht zuletzt darum, welche Erziehungsziele auf dem Weg zur*zum Staatsbürger*in realisiert werden sollen (Dreier 2018, S. 132): Diese changieren zwischen dem Ziel der Vermittlung von „Gottesfurcht“ oder einer bestimmten Glaubenswahrheit - und der damit verbundenen Vorstellungen von Körper, Geschlecht und Sexualität - und der strikten Neutralität eines Staates, der Glaubensinhalte und individuelle Überzeugungen nicht zum Gegenstand von Bevölkerungspolitik im Foucault'schen Sinne macht. Während die Förderung der Vermittlung bestimmter Glaubenswahrheiten spezifisch religiöse Identitätsangebote von Verfassungen stärken, stärken Lehrinhalte im Rahmen der staatlichen Neutralität verpflichteten Curricula das Identitätsangebot des in westlich-aufklärerischer Denktradition für sein Gewissen selbst verantwortlichen Individuums.

\subsection{Future citizens in Verfassungstexten}

Empirisch lässt sich diese Bandbreite in den Verfassungstexten wiederfinden. Nur in 44 Verfassungen $(23,6 \%)$ finden sich keinerlei Reglementierungen zum Schulunterricht; 46 Verfassungen verpflichten nur Staatsbürger*innen zum verbindlichen Schulunterricht, während 96 Verfassungen (51,6\%) eine allgemeine Schulpflicht für alle formulieren. Tab. 2 zeigt, dass darüber hinaus weit mehr als die Hälfte aller Verfassungen (105 Verfassungen) Regelungen zur religiösen Unterweisung umfassen; diese lassen sich nach verschiedenen Dimensionen unterscheiden. Hierzu gehören 
Tab. 2 Religiöse Unterweisung in den Verfassungstexten ${ }^{\mathrm{a}}$

\begin{tabular}{|c|c|c|}
\hline \multicolumn{2}{|l|}{ Art der Regelung } & Anzahl/Anteil \\
\hline \multicolumn{2}{|c|}{ Religiöse Unterweisung ist verfassungstextliches Thema } & $105(56,4 \%)$ \\
\hline \multicolumn{2}{|c|}{ Konfessionelle Schulen werden staatlich unterstützt } & $50(47,5 \%$ von 105$)$ \\
\hline \multirow[t]{5}{*}{ Religiöse Unterweisung } & ... liegt in der Verantwortung der Eltern & $49(46,6 \%$ von 105$)$ \\
\hline & ... ist nicht Sache des Staates & $26(24,7 \%$ von 105$)$ \\
\hline & Es gibt explizit keine religiöse Unterweisung & $15(14,2 \%$ von 105$)$ \\
\hline & Dem Staat ist die Intervention untersagt & $3(3 \%$ von 105$)$ \\
\hline & ... ist Angelegenheit des Staates & $37(35,2 \%$ von 105$)$ \\
\hline
\end{tabular}

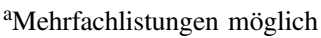

Quelle: RNCw - Eigene Berechnungen

die Dimension der Trägerschaft, der staatlichen Unterstützung, aber auch der Bestimmungshoheit über Curricula.

Von allen Verfassungen, die religiöse Erziehung als zu regelnden Tatbestand etablieren, überantworten 46,6\% (49 Verfassungen) die religiöse Unterweisung den Eltern oder Erziehungsberechtigten. Hier stärkt der Staat explizit die (gesellschaftlich als ,natürlich“ angesehenen) Elternrechte, zieht sich aus der Einheit der Familie zurück und verpflichtet die Eltern zur Pflege und Erziehung der Kinder. ${ }^{39}$

Verfassungen bestimmen auch darüber, ob und in welchem Maße sich der Staat curricular oder durch das Angebot von Religionsunterricht mittelbar in die Vermittlung von Glaubenswerten einmischen können soll. Die verfassungstextlichen Verankerungen reichen dabei von der Übernahme der umfassenden staatlichen Verantwortung für den Religionsunterricht und seine Inhalte bis zur vollständigen staatlichen Neutralität: Während beispielsweise die deutsche Verfassung dem Staat die Hoheit über den Religionsunterricht zuweist, ${ }^{40}$ verpflichtet die japanische Verfassung den Staat im Rahmen der Trennung von Staat und Religion auf vollständige Abstinenz bei der religiösen Unterweisung: „The State and its organs shall refrain from religious education or any other religious activity“ (Art. 20). Tab. 2 gibt einen Überblick über die Anzahl der Verfassungen, die den Staat auf die finanzielle und sonstige Unterstützung konfessioneller Schulen verpflichten und/oder religiöse Unterweisung regeln.

Von den 50 Verfassungen, in denen der Staat auf die finanzielle und sonstige Unterstützung konfessioneller Schulen verpflichtet werden, ist jedoch in 27 Verfassungen die religiöse Unterweisung explizit nicht Sache des Staates. In 19 dieser Fälle wird die Verantwortung für die religiöse Unterweisung explizit in die Hände der Eltern gelegt. Dieses Arrangement lässt sich als besondere Form der staatlichen Nichteinmischung lesen: Der Staat unterstützt Schulen in religiöser Trägerschaft in

\footnotetext{
39 Im deutschen Recht wird das Elternrecht beispielsweise durch den Art. 6 (3) GG festgelegt, der Eltern dieses als natürliches Recht zugesteht und die staatliche Gemeinschaft auf die Unterstützung der Familie festlegt (vgl. hierzu auch Czermak 2008, S. 65 und 136ff.).

40 So heißt es etwa in der deutschen Verfassung: „Der Religionsunterricht ist in den öffentlichen Schulen mit Ausnahme der bekenntnisfreien Schulen ordentliches Lehrfach. Unbeschadet des staatlichen Aufsichtsrechtes wird der Religionsunterricht in Übereinstimmung mit den Grundsätzen der Religionsgemeinschaften erteilt. Kein Lehrer darf gegen seinen Willen verpflichtet werden, Religionsunterricht zu erteilen“ (Art. 7 (3)).
} 
ihrem Erziehungsauftrag, interveniert aber nicht in die vermittelten Inhalte und belässt gleichzeitig die letzte Verantwortung für die Vermittlung von Weltanschauung bei den Eltern.

Von den 37 Verfassungen, in denen schulische Inhalte - und damit auch die religiöse Unterweisung - in den Verantwortungsbereich des Staates fallen, vergewissern sich 13 Verfassungen der Mitverantwortung der Eltern und 11 Verfassungen verpflichten den Staat auf finanzielle und organisatorische Unterstützung religiöser Schulen. Es lässt sich argumentieren, dass insbesondere in diesen 11 Fällen weit über die staatliche Neutralität hinaus die Biopolitik der Fabrikation der future citizens in den Verantwortungsbereich des Staates verlagert wird, indem der Staat auch in die Vermittlung religiöser Inhalte durch Schulen religiöser Gemeinschaften eingreift. Befürworten lässt sich ein solches Arrangement dadurch, dass staatlich unterstützte konfessionelle Schulen eine positive Antwort auf Rassismus seien, indem sie eine explizite Integration von Minderheiten bei gleichzeitiger Garantie einer schulischen Unterweisung auf dem Niveau der staatlichen (oder kommunalen) Schulen sicherstellten (Jackson 2003, S. 90f.). Dagegen lässt sich einwenden, dass solche Arrangements die Kontrolle religiöser Unterweisung ermöglichen. Hier käme der Staat seiner Ordnungsfunktion nach, indem er seine Souveränität gegen religiöse Autonomie von Religionsgemeinschaften und Eltern geltend macht.

Diese Variabilität in den verfassungstextlichen Verankerungen staatlicher Verantwortung für religiöse Unterweisung zeigt, dass im Feld der religiösen und weltanschaulichen Entwicklung der future citizens um die Verantwortlichkeiten, aber auch die Zugriffsmöglichkeiten von Staat, religiösen Gemeinschaften und Eltern gekämpft wird (Czermak 2008, S. 136). Mit Foucault ließe sich vermuten, dass es hier um nichts Geringeres geht als um die Vorherrschaft über die Wege der gesellschaftlichen Verankerung bestimmter ideologischer Deutungsmuster und deren normierender Kraft: Diese oszilliert, wie die unterschiedlichen Verankerungen zeigen, zwischen dem Schutz der Autonomie von Religionsgemeinschaften, wie diese z. B. in den USA gegen staatliche Eingriffe verteidigt wird (vgl. z. B. Carmella 1993), und dem Schutz von staatlicher Verfasstheit und seines Demos vor unerwünschten religiösen Weltanschauungen und religiösen Praxen. ${ }^{41}$

\section{Diskussion und Ausblick}

Der vorliegende Beitrag zeigt am Beispiel der verfassungstextlichen Verortung von Religion, auf welche Weisen Verfassungstexte als Komplex besonderer Rechtsvorschriften Identitätsangebote formulieren und dadurch ideale good citizens und future citizens bestimmen. Um dies zu zeigen, wurde auf den international vergleichenden Datensatz (RNCw, vgl. Schnabel et al. 2020) zurückgegriffen, der Verfassungen auf der Basis ihrer Textlichkeit quantitativ vergleichbar macht. Es konnte gezeigt werden, dass sich die verfassungstextlichen Identitätsangebote sowohl in den Präambeln als auch in den Regelungen zur individuellen Religionsfreiheit, die im Kontext des

\footnotetext{
41 Vgl. exemplarisch die Auseinandersetzung um das Tragen von Kopftüchern von Schülerinnen und Leh-
} rerinnen (z. B. Benhabib 2010). 
verfassungsmäßig spezifizierten Staat-Religions-Verhältnisses gelesen wurde, sowie in den Regelungen zur religiösen Unterweisung finden lassen.

Die hier vorgelegten Analysen abstrahieren vollständig von der aktuell in einem Land realisierten Regierungsform. Dies wird dadurch begründet, dass hier allein die Subjektformation in Verfassungstexten, nicht aber ihre Realisierung im Fokus stand. Die Analyse bestätigt, was bereits Go (2003, S. 90) feststellte: „Thus, if a globalizing constitutionalism has emerged at all, it is one wracked with divergence as much as convergence, a differentiation in content as much as a homogenization in form“. Religion bleibt einer derjenigen Identitätsmarker, die in Verfassungstexten pfadabhängig verankert sind.

Neben Ähnlichkeiten in der Form $(83,9 \%$ der Verfassungen haben eine Präambel) finden sich Ähnlichkeiten in den Dimensionen: Mehr als $95 \%$ der Verfassungen beinhalten das Versprechen auf individuelle Religionsfreiheit, knapp 80\% der Verfassungstexte enthalten Regelungen zum Staat-Religions-Verhältnis; verfassungstextliche Regelungen zur religiösen Unterweisung finden sich immer noch in weit mehr als der Hälfte der Verfassungstexte (56\%). Auch wenn diese Dimensionen unterschiedlich ausgestaltet sind, zeigt dies, dass staatliche Gemeinschaften Religion nach wie vor als einen so wichtigen Tatbestand ansehen, dass er im grundlegendsten Normsetzungsdokument geregelt werden soll. Damit erlangt dieser Tatbestand aber nicht nur für die Mitglieder des Demos Relevanz, wenn sie sich in unterschiedlichen Praktiken des Befolgens, Ignorierens, Umkämpfens oder Klage Einreichens dazu verhalten; sie werden hier mit Bezug zur Religion im engen Wortsinn regelrecht konstituiert: Die textliche Anrufung lässt sich als idealisiertes Bild des good citizen lesen, welches das Identitätsangebot für die Bürger*innen bestimmt.

Je nach Verankerung umfasst diese Konstituierung das Recht auf vollständig individualisierte Bezugnahme auf Religion, wenn der Staat sich dem Neutralitätsgebot verpflichtet und seine Bürger*innen als Individuen thematisiert, die zu freien Gewissensentscheidungen fähig sind. Das andere Ende des Spektrums bilden Verfassungen mit verbindlichen Identitätsangeboten in Form von Staatsreligionen. Diese werden dann in der Verfassungswirklichkeit in unterschiedlicher Form eingefordert und können in ihrer rigiden Form zu Benachteiligungen und Repressionen gegen Bevölkerungsgruppen führen, die diesem Identitätsangebot nicht entsprechen. Wie andere Identitäten auch, ${ }^{42}$ sind auch verfassungstextliche Identitätsangebote exklusiv, indem sie Grenzen markieren, die sich empirisch in Diskriminierung und Anfeindung manifestieren können. So kommt der PEW-Report (2019) zu dem Ergebnis, dass ,[o]ver the decade from 2007 to 2017, government restrictions on religion - laws, policies and actions by state officials that restrict religious beliefs and practices increased markedly around the world. And social hostilities involving religion - including violence and harassment by private individuals, organizations or groups also have risen since 2007.“ Der Bericht zählt für 2017 insgesamt 52 Länder mit staatlicher Repression gegen religiöse Gemeinschaften.

Über das Auseinanderfallen von Verfassungstext und Verfassungswirklichkeit hinaus eröffnen viele Verfassungen intratextliche Optionen, die individuelle Ausübung der Religionsfreiheit zugunsten des Schutzes der Verfassungsordnung und des De-

42 Vgl. z. B. Jenkins (2004, S. 102 ff.) oder Lamont (2001, S. 172 ff.). 
mos zu beschränken. Hier offenbart sich eine Form der institutionellen Absicherung der staatlichen Ordnung, die (bestimmten) Bürger*innen qua abweichender religiöser Identität Anreize zur Nichtbefolgung dieser Ordnung unterstellt. Es findet sich hier das, was Agamben (2017, S. 51) als den „Ausnahmezustand“ diskutiert: „Die Eröffnung eines Raums, in dem Anwendung und Norm ihre Getrenntheit zur Schau stellen und worin reine Gesetzeskraft eine Norm umsetzt (sprich: sie anwendet indem sie sie nicht anwendet)." Dies wird nicht zuletzt darin deutlich, dass keiner der vorliegenden Verfassungstexte genauer bestimmt, was unter ,public order“ oder „public morals“ verstanden werden soll. Verfassungen grenzen damit nicht nur die Macht des staatlichen Souveräns zugunsten seines Demos ein, sondern schützen ihn auch vor der Religiosität eben dieses Demos.

Verfassungstexte entwerfen aber nicht nur den aktuell gültigen Demos, sie fixieren ihn für die Zukunft: Indem Verfassungen die Verantwortung religionsbezogener und weltanschaulicher Unterweisung zwischen Eltern, staatlichen und konfessionellen oder religionsgemeinschaftlichen Schulen aufteilen, verteilen sie auch das Recht auf die inhaltliche Ausgestaltung von Erziehung und damit auf den Zugriff auf die religiöse und weltanschauliche Orientierung zukünftiger Generationen.

Verfassungen sind damit soziologisch relevant: Sie ordnen Gesellschaften nicht nur rechtlich, sondern auch, indem sie die staatlichen Bevölkerungen verfassungsidentitär bestimmen und normieren. Über die rechtlichen Artikel hinaus umfassen sie eine Verfassungsidentität und konstituieren damit den Demos auch in einer außerrechtlichen, alltäglich praktischen Form. Die Verfassungsidentität ist dabei einerseits Ausdruck internationaler Diskurse - wie dem Diskurs zur individuellen Religionsfreiheit, der sich in fast allen Verfassungen spiegelt (z. B. Thornhill 2017, S. 497) als auch pfadabhängiger landesspezifischer Historien (z. B. Go 2003).

Die bisherigen Ausführungen machten von einem Datensatz Gebrauch, der auf der Basis einer quantifizierenden Inhaltsanalyse Verfassungstexte international vergleichbar macht. Dabei ging es darum, Ähnlichkeiten und Differenzen in den Verfassungstexten aufzuspüren und diese über die Zuweisung von Zahlenwerten vergleichbar zu machen. Solche Vergleiche sind notgedrungen immer limitiert: Sie können nur einer rein textbasierten Basis folgen und müssen länderspezifische juristische Auslegungen auch gleicher Formulierungen ebenso vernachlässigen wie ihren Entstehungskontext oder die Verfassungswirklichkeit. Insbesondere die im soziologischen Konstitutionalismus thematisierten Isomorphismen (Boli-Bennett 1979) und Pfadabhängigkeiten der historischen und aktuellen Entwicklung (Culic 2003; Thornhill 2011) müssen unbeachtet bleiben. Um diese näher zu untersuchen, werden entsprechende tiefenanalytische Fallstudien benötigt (wie z.B. von Witte und Bucholc 2016).

Auch die länderspezifische Rechtsprechung spielte hier keine Rolle, weil sich diese zwischen Ländern nur eingeschränkt vergleichen lässt. Dennoch ist Rechtsprechung wesentlich, um zu verstehen, wie Verfassungstexte vor dem Hintergrund aktueller Entwicklungen ausgelegt und angewandt werden: ${ }^{43}$ Verfassungstexte sind Momentaufnahmen bestimmter Machtkonstellationen - wenn sie verfasst sind, müs-

${ }^{43}$ Dreier (2018, S. $146 \mathrm{ff}$. ) verweist in diesem Zusammenhang darauf, dass Verfassungstexte eben keine sakralen Texte sind und eben nicht mystifiziert und als Offenbarung gelesen werden dürften. 
sen sie mit Bezug zu aktuellen Entwicklungen, Ereignissen und Problemstellungen angewandt und ausgelegt werden, da weder alle Zeitläufe im Vorhinein geregelt werden können, noch dies gewollt sein kann. Die Zusammensetzung des Demos verändert sich nicht zuletzt durch Migration, Bildungsexpansion oder die Entwicklung des Gesundheitssystems; technische Herausforderungen stellen bisherige Wertsetzungen in Frage. Nicht immer wird solchen Veränderungen mit einer Neufassung der Verfassung begegnet.

Mit ihren Identitätsangeboten konfigurieren Verfassungen good citizenship und schaffen damit nicht nur eine rechtsstaatliche, sondern auch eine soziale Ordnung, indem Bürger*innen als Subjekte mit Rechten und Pflichten angerufen werden. Im globalen Vergleich bleibt Religion dabei wichtiges Element - sei es als Adresse der staatlichen Schutzverpflichtung individueller Freiheiten, als Moment der Bedrohung der Freiheit anderer und der staatlichen Ordnung oder als konfessionell bestimmter Bestandteil der staatlichen Gemeinschaft. Die Stabilität dieser Konfigurationen hängt nicht zuletzt davon $\mathrm{ab}$, inwieweit die Bürger*innen ihre verfassungstextliche Verfasstheit in ihren Einstellungen und alltäglichen und politischen Praxen zu reproduzieren bereit sind. Davon hängt auch die Aufrechterhaltung des verfassungstextlich fixierten gesellschaftlichen Konsenses ab - der eben nicht zuletzt auch die Freiheit von und zur Religion und zur gegenseitigen Anerkennung umfasst.

\section{Anhang}

Tab. 3 Zusammenhang zwischen kollektivem ,wir“ und Gottes- oder Religionsbezug

\begin{tabular}{|c|c|c|c|c|c|c|}
\hline & & $\begin{array}{l}\text { Keine } \\
\text { Präam- } \\
\text { bel }\end{array}$ & $\begin{array}{l}\text { Kein Gottes-/ } \\
\text { Religionsbezug }\end{array}$ & $\begin{array}{l}\text { Verpflichtung } \\
\text { zur Säkularität }\end{array}$ & $\begin{array}{l}\text { Gottes-/ } \\
\text { Religionsbezug }\end{array}$ & $\begin{array}{l}\text { Ge- } \\
\text { samt }\end{array}$ \\
\hline \multirow{3}{*}{$\begin{array}{l}\text { Präambel } \\
\text { im Namen } \\
\text { des } \\
\text { kollektiven } \\
\text { „wir“" } \\
\text { formuliert }\end{array}$} & $\begin{array}{l}\text { Keine } \\
\text { Präambel }\end{array}$ & 30 & 0 & 0 & 0 & 30 \\
\hline & $\begin{array}{l}\text { Nicht } \\
\text { erwähnt }\end{array}$ & 0 & 36 & 3 & 51 & 90 \\
\hline & $\begin{array}{l}\text { „We, the } \\
\text { people .... }\end{array}$ & 0 & 24 & 6 & 36 & 66 \\
\hline Gesamt & & 30 & 60 & 9 & 87 & 186 \\
\hline
\end{tabular}

Quelle: $\mathrm{RNCw}$ - Eigene Berechnungen 
Danksagung Unter Mitarbeit von Nediem Abdulla Arem, Kathrin Behrens und Heiko Beyer; ihnen gebührt an dieser Stelle besonderer Dank.

Funding Open Access funding enabled and organized by Projekt DEAL.

Open Access Dieser Artikel wird unter der Creative Commons Namensnennung 4.0 International Lizenz veröffentlicht, welche die Nutzung, Vervielfältigung, Bearbeitung, Verbreitung und Wiedergabe in jeglichem Medium und Format erlaubt, sofern Sie den/die ursprünglichen Autor(en) und die Quelle ordnungsgemäß nennen, einen Link zur Creative Commons Lizenz beifügen und angeben, ob Änderungen vorgenommen wurden.

Die in diesem Artikel enthaltenen Bilder und sonstiges Drittmaterial unterliegen ebenfalls der genannten Creative Commons Lizenz, sofern sich aus der Abbildungslegende nichts anderes ergibt. Sofern das betreffende Material nicht unter der genannten Creative Commons Lizenz steht und die betreffende Handlung nicht nach gesetzlichen Vorschriften erlaubt ist, ist für die oben aufgeführten Weiterverwendungen des Materials die Einwilligung des jeweiligen Rechteinhabers einzuholen.

Weitere Details zur Lizenz entnehmen Sie bitte der Lizenzinformation auf http://creativecommons.org/ licenses/by/4.0/deed.de.

\section{Literatur}

\section{Verwendete Literatur}

Addis, A. 2018. Constitutional preambles as narratives of peoplehood. Vienna Journal of International Constitutional Law 12(2):125-181.

Agamben, G. 2017. Ausnahmezustand. Frankfurt a.M.: Suhrkamp.

Alexander, L. 1998. Constitutionalism. Philosophical foundations. Cambridge: Cambridge University Press.

Anderson, B. 1988. Die Erfindung der Nation. Frankfurt, New York: Campus.

Behrens, K. 2021. Formal citizenship in European constitutions. In Democratic citizenship in flux, Hrsg. M. Bayer, O. Schwarz, und T. Stark, 83-100. Bielefeld: transcript.

Benhabib, S. 2010. The return of political theology: the scarf affair in comparative constitutional perspective in France, Germany and Turkey. Philosophy \& Social Criticism 36(3-4):451-471.

Beyer, H. 2018. Religion als Menschenrecht: Zur paradoxen Struktur des Rechts auf Religionsfreiheit. In Religion im Kontext: Handbuch für Wissenschaft und Studium, Hrsg. A. Schnabel, M. Reddig, und H. Winkel, 239-255. Baden-Baden: Nomos.

Blaustein, A.P. 1994. Framing the modern constitution: a checklist. A Philadelphia constitution book. Littleton: Rothman \& Co.

Bock, W. 1998. Die Religionsfreiheit zwischen Skylla und Charybdis. Archiv des öffentlichen Rechts 123(3):444-475.

Böckenförde, E.-W. 1989. Recht, Staat, Europa. Frankfurt, a.M: Suhrkamp.

Böckenförde, E.-W. 2011. Wissenschaft, Politik, Verfassungsgericht. Berlin: Suhrkamp.

Boli-Bennett, J. 1979. The ideology of expanding state authority in national constitutions, 1870-1970. In National development and the world system: educational, economic and political change, 1950-1970, Hrsg. J.W. Meyer, M.T. Hannan, 222-249. Chicago: University of Chicago Press.

Butler, J. 1988. Performative acts and gender constitution. Theatre Journal 40(4):519-531.

Carmella, A. 1993. State constitutional protection of religious exercise: an emerging post-smith jurisprudence. Bуи Law Review 275:275-327.

Culic, I. 2003. State building and constitution writing in central and eastern Europe after 1989. RegioMinorities, Politics, Society 1:38-58.

Czermak, G. 2008. Religions- und Weltanschauungsrecht. Berlin, Heidelberg: Springer.

Dreier, H. 2018. Staat ohne Gott. München: Beck.

Van Dülmen, R. 2016. Die Entdeckung des Individuums: 1500-1800. Frankfurt a.M.: Fischer.

Elkins, Z., T. Ginsburg, und J. Melton. 2009. The endurance of national constitutions. Cambridge: University Press. 
Elkins, Z., T. Ginsburg, und J. Melton. 2014. Characteristics of national constitutions, v.2.0. https:// comparativeconstitutionsproject.org. Zugegriffen: 16. März 2021.

Foucault, M. 1978. The history of sexuality. New York: Pantheon.

Fox, J. 2014. The religion and state project. https://www.thearda.com/ras. Zugegriffen: 16. März 2021.

Gamper, A. 2012. Regeln der Verfassungsinterpretation. Wien: Springer.

Gardbaum, S. 2012. The place of constitutional law in the legal system. In The oxford handbook of comparative constitutional law, Hrsg. M. Rosenfeld, A. Sajó, 169-185. Oxford: Oxford University Press.

Garfinkel, H. 1967. Studies in Ethnomethodology. Cambridge: Blackwell.

Glaeßner, G.-J. 1997. Von der Perestroika zur liberalen Demokratie? Strukturprobleme des Systemwechsels und der Demokratisierung im Postkommunismus. In Systemwechsel und Demokratisierung, Hrsg. G.-J. Glaeßner, M. Reiman, 13-43. Opladen: Westdeutscher Verlag.

Go, J. 2003. A globalizing constitutionalism? Views from the Postcolony, 1945-2000. International Sociology 18:71-95.

Greenawalt, K. 2008. Religion and the constitution. Princeton: Princeton University Press.

Grey, T.C. 1984. The constitution as scripture. Stanford Law Review 37:1-25.

Grimm, D. 2008. Conflicts between general laws and religious norms. Cardozo Law Review 30:2369-2382.

Häberle, P. 1982. Präambeln im Text und Kontext von Verfassungen. In Demokratie in Anfechtung und Bewährung. Festschrift für Johannes Broemann, Hrsg. J. Listl, H. Schambeck, 211-249. Berlin: Duncker \& Humblot.

Häberle, P. 1998. Verfassungslehre als Kulturwissenschaft. Berlin: Dunker \& Humblot.

Heintz, B., und A. Schnabel. 2006. Verfassungen als Spiegel globaler Normen? Kölner Zeitschrift für Soziologie und Sozialpsychologie 58(4):685-716.

Jackson, R. 2003. Should the state fund Faith based schools? British Journal of Religious Education 25(2):89-102.

Jacobsohn, G.J. 2006. Constitutional identity. The Review of Politics 68(3):361-397.

Jenkins, R. 2004. Social identity. London: Routledge.

Jones, F.L., und P. Smith. 2001. Individual and sociental bases of national identity. European Sociological Review 17:103-118.

Kelsen, H. 1946. The preamble of the charter. A critical analysis. The Journal of Politics 8(2):134-159.

Klein, C., und A. Sajó. 2012. Constitution-making: process and substance. In The oxford handbook of comparative constitutional law, Hrsg. M. Rosenfeld, A. Sajó, 419-441. Oxford: Oxford University Press.

Kymlicka, W., und W. Norman. 1994. Return of the citizen: a survey of recent work on citizenship theory. Ethics 104(2):352-381.

Lamont, M. 2001. Culture and identity. In Handbook of sociological theory. Handbooks of sociology and social research, Hrsg. J.H. Turner, 171-185. Boston: Springer.

Lindemann, G. 2009. Gesellschaftliche Grenzregime und soziale Differenzierung. Zeitschrift für Soziologie 38(2):94-112.

Luhmann, N. 1990. Verfassung als evolutionäre Errungenschaft. Rechtshistorisches Journal 9:177-220.

Lutz, D. 2000. Thinking about constitutionalism at the start of the twenty-first century. Publius: The Journal of Federalism 30:115-135.

Nussbaum, M. 2008. Liberty of conscience. New York: Basic Books.

Öhlinger, T., und H. Eberhard. 2019. Verfassungsrecht. Wien: fakultas.

Orgad, L. 2010. The preamble in constitutional interpretation. International Journal of Constitutional Law $8(4): 714-738$.

PEW. 2019. A closer look at how religious restrictions have risen around the world. PEW-forum. https:// www.pewforum.org/2019/07/15/a-closer-look-at-how-religious-restrictions-have-risen-aroundthe-world/ (Erstellt: 15. Juli 2019). Zugegriffen: 2. Aug. 2020.

Pykett, J., M. Saward, und A. Schaefer. 2010. Framing the good citizen. The British Journal of Politics and International Relations 12(4):523-538.

Reuter, A. 2007. Säkularität und Religionsfreiheit - ein doppeltes Dilemma. Leviathan 35(2):178-192.

Robertson, R. 1990. After nostalgia? Willful nostalgia and the phase of globalization. In Theories of modernity and postmodernity, Hrsg. B.S. Turner, 45-61. London: SAGE.

Rosenfeld, M. 2009. The identity of the constitutional subject: selfhood, citizenship, culture, and community. Abingdon: Routledge.

Rosenfeld, M. 2012. Constitutional Identity. In The oxford handbook of comparative constitutional law, Hrsg. M. Rosenfeld, A. Sajó, 756-777. Oxford: University Press.

Rosenfeld, M. und A. Sajó. 2012. The Oxford Handbook of Comparative Constitutional Law. Oxford: Oxford University Press. 
Sajó, A., und R. Uitz. 2012. Freedom of religion. In The oxford handbook of comparative constitutional law, Hrsg. M. Rosenfeld, A. Sajó, 909-928. Oxford: Oxford University Press.

Schmidt, R. 2012. Verfassungskultur und Verfassungssoziologie. Wiesbaden: Springer.

Schnabel, A., K. Behrens, und F. Grötsch. 2017. Religion in European constitutions-cases of different secularities. European Societies 19(5):551-579.

Schnabel, A., H. Beyer, und K. Behrens. 2020. Religious freedom, equality rights and their contentious implementation: norm conflicts deriving from the chasm between international and national human rights. In Religion, migration and existential wellbeing, Hrsg. M. Kindström Dahlin, O. Larsson, und A. Winell, 21-39. London: Routledge.

Thornhill, C. 2011. A sociology of constitutions. Cambridge: Cambridge University Press.

Thornhill, C. 2017. The sociology of constitutions. Annual Review of Law and Social Science 13:493-515.

Tschentscher, A. 2011. Comparing constitutions and international constitutional law: a primer. http://ssrn. com/abstract=1502125. Zugegriffen: 2. Aug. 2020. https://doi.org/10.2139/ssrn.1502125.

Tushnet, M. 1983. Constitution-making: an introduction. Texas Law Review 91:1983-2013.

Tushnet, M. 2010. How do constitutions constitute constitutional identity? International Journal of Constitutional Law 8:671-676.

Vorländer, H. 2002. Integration durch Verfassung., 9-40. Wiesbaden: VS.

Vorländer, H. 2017. Constitutions as symbolic orders: the cultural analysis of constitutionalism. In Sociological constitutionalism, Hrsg. P. Blokker, C. Thornhill, 209-240. Cambridge: Cambridge University Press.

Waluchow, Wil. 2018. Constitutionalism. In The stanford encyclopedia of philosophy, Hrsg. E.N. Zalta https://plato.stanford.edu/archives/spr2018/entries/constitutionalism; letzter Aufruf 11.03.2021.

Witte, D. und M. Bucholc (2016): Verfassungsanalyse als (rechts-)kulturvergleichende Methode: Theoretische Perspektiven und das Beispiel der polnischen Verfassungskrise. In: S. Lessenich (Hrsg.): Geschlossene Gesellschaften. Verhandlungen des 38. Kongresses der Deutschen Gesellschaft für Soziologie [http://publikationen.soziologie.de/index.php/kongressband_2016/article/view/547, letzter Aufruf 11.03.2021]

\section{Quelle}

Schnabel, A., K. Behrens, L. Hönes, und H. Beyer. 2020. Religion and Nation in Constitutions Worldwide $(R N C w)$ (Version: 1.0.0). https://doi.org/10.7802/1991. 